Supporting Information

For

\title{
A Highly Efficient and Selective Route to Isomeric Cyclic Diazadienes
}

\author{
Mark E. Scott, Yann Bethuel and Mark Lautens ${ }^{*}$ \\ Davenport Research Laboratories, Department of Chemistry, University of Toronto, \\ 80 St. George Street, Toronto, Ontario, M5S 3H6, Canada \\ mlautens@chem.utoronto.ca
}




\section{Table of Contents}

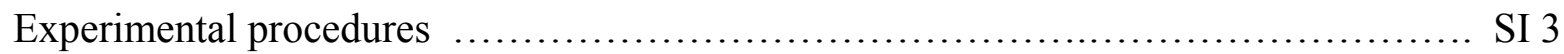

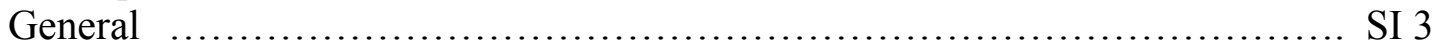

Starting Material Synthesis ….......................................... SI 3

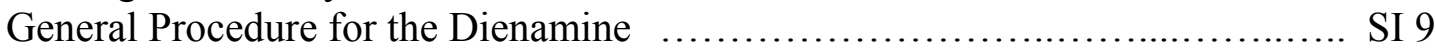

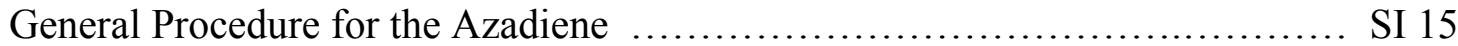

Mechanistic Studies ...................................................................................... SI 16

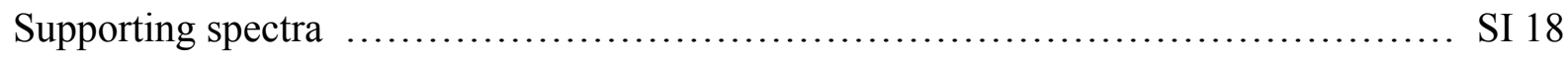

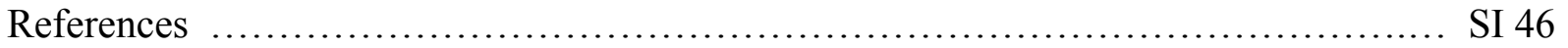




\section{General}

Melting points were recorded using a Fisher-Johns melting point apparatus and are uncorrected. ${ }^{1} \mathrm{H}$ and ${ }^{13} \mathrm{C}$ NMR spectra were recorded using either a Varian Mercury $400 \mathrm{MHz}$ or Varian Unity $500 \mathrm{MHz}$ spectrometer in $\mathrm{CDCl}_{3}$ with chemical shifts relative to tetramethylsilane (TMS, $0 \mathrm{ppm}$ ) unless otherwise reported. No special notation is used for equivalent carbons. IR spectra were obtained using a Nicolet DX FT IR spectrometer as thin films on $\mathrm{NaCl}$ plates. High-resolution mass spectra were obtained using either a VG 70-250S (double focusing) mass spectrometer at $70 \mathrm{eV}$ or a Sciex Qstar turbo ionspray mass spectrometer.

Diethyl ether and dichloromethane were dried and degassed using an MBRAUN MB solvent purification system. Tetrahydrofuran (THF) was distilled from Na/benzophenone prior to use. Dimethoxyethane was distilled from $\mathrm{CaH}_{2}$ prior to use. All other reagents were used as received unless otherwise indicated. $\mathrm{MgI}_{2}$ was weighed out in a glovebox to prevent the absorption of water. Neutral silica (Silicycle, Quebec, Canada) for flash chromatography was used as received. All experiments were performed under anhydrous conditions under an atmosphere of argon. 2-Methylene-cyclopropanecarboxylic acid, ${ }^{1}$ dideuteriodiiodomethane ${ }^{2}$ and buta-2,3-dien$1-\mathrm{ol}^{3}$ were prepared as previously reported.

\section{Starting Material Synthesis}

2-Methylene-cyclopropanecarboxylic acid methoxy-methyl-amide

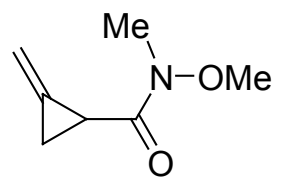

To a solution of 2-methylene-cyclopropanecarboxylic acid $(7.16 \mathrm{~g}, 73.0 \mathrm{mmol})$ in dichloromethane $(372 \mathrm{~mL})$ at $0{ }^{\circ} \mathrm{C}$ was added carbonyldiimidizole $(14.1 \mathrm{~g}, 87.1 \mathrm{mmol})$. The resulting solution was stirred for 1 hour at $0{ }^{\circ} \mathrm{C}$. To this solution was added $O, N$-dimethylhydroxylamine hydrochloride $(17.8 \mathrm{~g}, 0.183 \mathrm{~mol})$ and the reaction was stirred overnight. The reaction solution was then filtered through a bed of Celite ${ }^{\circledR}$ and the filtrate was concentrated in vacuo. Flash chromatography on silica using 2:3 diethyl ether:hexanes $\left(R_{f}=0.21\right)$ afforded the title compound $\left(8.96 \mathrm{~g}, 87 \%\right.$ ) as a colourless oil. IR (neat, $\left.\mathrm{cm}^{-1}\right) 3565,2975,2938,1654,1458$, $1418,1385,1179,1110,988,908,890,736,686 ; \delta_{\mathrm{H}}\left(400 \mathrm{MHz}, \mathrm{CDCl}_{3}\right) 5.45(\mathrm{~m}, 1 \mathrm{H}), 5.41(\mathrm{~m}$, $1 \mathrm{H}), 3.78(\mathrm{~s}, 3 \mathrm{H}), 3.23(\mathrm{~s}, 3 \mathrm{H}), 2.80(\mathrm{~m}, 1 \mathrm{H}), 1.88(\mathrm{~m}, 1 \mathrm{H}), 1.60$ (apparent tt, $1 \mathrm{H}, J=8.6,2.4$ $\mathrm{Hz}) ; \delta_{\mathrm{C}}\left(100 \mathrm{MHz}, \mathrm{CDCl}_{3}\right) 171.9,131.7,104.0,61.9,32.8,16.0,10.6 ; \mathrm{C}_{7} \mathrm{H}_{11} \mathrm{NO}_{2}\left(\mathrm{M}^{+}\right)(\mathrm{EI})$ Requires: 141.0790 Found: 141.0793. 


\section{1-(2-Methylene-cyclopropyl)-ethanone-tosyl-hydrazone}

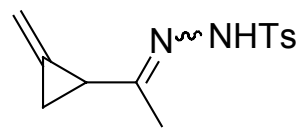

To a solution of 2-methylene-cyclopropanecarboxylic acid methoxy-methyl-amide $(0.268 \mathrm{~g}, 1.90$ $\mathrm{mmol})$ in diethyl ether $(10.3 \mathrm{~mL})$ at $-78{ }^{\circ} \mathrm{C}$ was added a solution of $\mathrm{MeMgBr}$ in diethyl ether (3.2 $\mathrm{M}, 0.66 \mathrm{~mL}, 2.11 \mathrm{mmol})$. The resulting white suspension was then stirred to room temperature for 4 hours until the reaction was complete. To this solution was added a $1 \mathrm{~N} \mathrm{HCl}$ solution (10 $\mathrm{mL})$. The reaction solution was then extracted with diethyl ether $(4 \times 50 \mathrm{~mL})$ and the combined organic layers were washed with brine, dried over $\mathrm{Na}_{2} \mathrm{SO}_{4}$, filtered and concentrated in vacuo. The crude product was then taken up in dichloromethane $(9.5 \mathrm{~mL}, 0.2 \mathrm{M})$ and $\mathrm{Na}_{2} \mathrm{SO}_{4}$ and $p$ tosylhydrazine $(0.355 \mathrm{~g}, 1.91 \mathrm{mmol})$ were added. The resulting solution was stirred at room temperature overnight and was then filtered and concentrated in vacuo. Flash chromatography on silica using 1:2 ethyl acetate:hexanes $\left(R_{f}=0.47\right)$ afforded the title compound $(0.375 \mathrm{~g}, 75 \%)$ as a white solid and as a 3:1 mixture of isomers. IR (neat, $\mathrm{cm}^{-1}$ ) 3216, 2988, 1620, 1598, 1396, $1337,1314,1166,1091,1059,1018,900,815,682 ; \delta_{\mathrm{H}}\left(400 \mathrm{MHz}, \mathrm{CDCl}_{3}\right) 7.87(\mathrm{~d}, 1.5 \mathrm{H}, J=8.1$ $\mathrm{Hz}), 7.86(\mathrm{~d}, 0.5 \mathrm{H}, J=8.1 \mathrm{~Hz}), 7.75(\mathrm{bs}, 1 \mathrm{H}), 7.37(\mathrm{bs}, 1 \mathrm{H}), 7.32(\mathrm{~d}, 2 \mathrm{H}, J=8.3 \mathrm{~Hz}), 5.53(\mathrm{~m}$, $0.25 \mathrm{H}), 5.51(\mathrm{~m}, 0.75 \mathrm{H}), 5.48(\mathrm{~m}, 0.25 \mathrm{H}), 5.45(\mathrm{~m}, 0.75 \mathrm{H}), 2.44(\mathrm{~s}, 3 \mathrm{H}), 2.39(\mathrm{~m}, 0.75 \mathrm{H}), 2.28$ $(\mathrm{m}, 0.25 \mathrm{H}), 1.70(\mathrm{~s}, 0.75 \mathrm{H}), 1.69(\mathrm{~m}, 0.25 \mathrm{H}), 1.50(\mathrm{~m}, 0.75 \mathrm{H}), 1.50(\mathrm{~s}, 3 \mathrm{H}), 1.32(\mathrm{~m}, 1 \mathrm{H}) ; \delta_{\mathrm{C}}$ $\left(125 \mathrm{MHz}, \mathrm{CDCl}_{3}\right)$ 157.8, 157.7, 144.8, 144.2, 135.7, 133.8, 131.7, 131.6, 130.2, 129.9, 129.7, 128.5, 128.4, 128.3, 106.4, 106.1, 104.7, 27.8, 27.3, 23.2, 21.8, 19.8, 15.5, 12.6, 11.6, 10.4, 9.6; $\mathrm{C}_{13} \mathrm{H}_{16} \mathrm{~N}_{2} \mathrm{O}_{2} \mathrm{~S}\left(\mathrm{M}^{+}\right)$(EI) Requires: 264.0933 Found: 264.0937.

\section{1-(2-Methylene-cyclopropyl)-propan-1-one-tosyl-hydrazone}

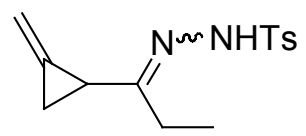

To a solution of 2-methylene-cyclopropanecarboxylic acid methoxy-methyl-amide $(0.228 \mathrm{~g}, 1.62$ mmol) in diethyl ether $(9.6 \mathrm{~mL})$ at $-78{ }^{\circ} \mathrm{C}$ was added a solution of $\mathrm{EtMgCl}$ in diethyl ether $(3.0$ $\mathrm{M}, 0.80 \mathrm{~mL}, 2.40 \mathrm{mmol})$. The resulting white suspension was stirred at room temperature for 7 hours until the reaction was complete. To this solution was added a $1 \mathrm{~N} \mathrm{HCl}$ solution $(10 \mathrm{~mL})$. The reaction solution was then extracted with diethyl ether $(4 \times 50 \mathrm{~mL})$ and the combined organic layers were washed with brine, dried over $\mathrm{Na}_{2} \mathrm{SO}_{4}$, filtered and concentrated in vacuo. The crude product was taken up in dichloromethane $(8.1 \mathrm{~mL}, 0.2 \mathrm{M})$ and $\mathrm{Na}_{2} \mathrm{SO}_{4}$ and $p$-tosylhydrazine $(0.452 \mathrm{~g}, 2.42 \mathrm{mmol})$ were added. The resulting solution was stirred at room temperature for one week and was then filtered and concentrated in vacuo. Flash chromatography on silica using 1:2 ethyl acetate:hexanes $\left(R_{f}=0.6-0.5\right)$ afforded the title compound $(0.390 \mathrm{~g}, 87 \%$ over two steps $)$ as a white solid and as a 1.9:1 mixture of isomers. IR (neat, $\mathrm{cm}^{-1}$ ) 3216, 2977, 1620, 1598, 1454, 1402 , 1330, 1165, 1091, 1020, 890, 814, 668; $\delta_{\mathrm{H}}\left(400 \mathrm{MHz}, \mathrm{CDCl}_{3}\right) 7.86(\mathrm{~d}, 1.3 \mathrm{H}, J=8.6 \mathrm{~Hz})$, $7.84(\mathrm{~d}, 0.7 \mathrm{H}, J=8.6 \mathrm{~Hz}), 7.74(\mathrm{bs}, 0.65 \mathrm{H}), 7.40(\mathrm{bs}, 0.35 \mathrm{H}), 7.31(\mathrm{~d}, 2 \mathrm{H}, J=7.8 \mathrm{~Hz}), 5.53(\mathrm{~m}$, $0.65 \mathrm{H}), 5.50(\mathrm{~m}, 0.65 \mathrm{H}), 5.47(\mathrm{~m}, 0.35 \mathrm{H}), 5.42(\mathrm{~m}, 0.35 \mathrm{H}), 2.43(\mathrm{~s}, 3 \mathrm{H}), 2.30(\mathrm{~m}, 0.35 \mathrm{H}), 2.15$ $(\mathrm{m}, 0.65 \mathrm{H}), 2.10-1.84(\mathrm{~m}, 2 \mathrm{H}), 1.67$ (apparent tt, 0.65H, $J=9.2,2.3 \mathrm{~Hz}), 1.48$ (apparent tt, $0.35 \mathrm{H}, J=9.2,2.3 \mathrm{~Hz}), 1.40(\mathrm{~m}, 0.35 \mathrm{H}), 1.22(\mathrm{~m}, 0.65 \mathrm{H}), 1.01(\mathrm{t}, 1.05 \mathrm{H}, J=7.8 \mathrm{~Hz}), 0.98(\mathrm{t}$, $1.95 \mathrm{H}, J=7.4 \mathrm{~Hz}) ; \delta_{\mathrm{C}}\left(100 \mathrm{MHz}, \mathrm{CDCl}_{3}\right) 161.2,159.6,144.1,135.6,131.9,129.7,129.6,128.3$, 
128.3, 106.3, 105.5, 27.1, 21.8, 21.8, 20.1, 14.8, 10.7, 10.4, 10.1, 9.6; $\mathrm{C}_{14} \mathrm{H}_{19} \mathrm{~N}_{2} \mathrm{O}_{2} \mathrm{~S}\left(\mathrm{MH}^{+}\right)(\mathrm{ESI})$ Requires: 279.1161 Found: 279.1150.

\section{1-(2-Methylene-cyclopropyl)-2-phenyl-ethanone-tosyl-hydrazone}

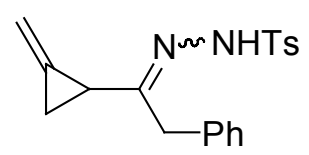

To a solution of 2-methylene-cyclopropanecarboxylic acid methoxy-methyl-amide $(0.125 \mathrm{~g}$, $0.885 \mathrm{mmol})$ in diethyl ether $(4.8 \mathrm{~mL})$ at $-78{ }^{\circ} \mathrm{C}$ was added a solution of $\mathrm{BnMgBr}$ in diethyl ether (0.6 M, $1.62 \mathrm{~mL}, 0.972 \mathrm{mmol})$. The resulting solution was then stirred to room temperature for 1.5 days. To this solution was added a $1 \mathrm{~N} \mathrm{HCl}$ solution $(10 \mathrm{~mL})$. The reaction solution was extracted with diethyl ether $(4 \times 50 \mathrm{~mL})$ and the combined organic layers were washed with brine, dried over $\mathrm{Na}_{2} \mathrm{SO}_{4}$, filtered and concentrated in vacuo. The crude product was taken up in dichloromethane $(4.5 \mathrm{~mL}, 0.2 \mathrm{M})$ and $\mathrm{Na}_{2} \mathrm{SO}_{4}$ and $p$-tosylhydrazine $(0.248 \mathrm{~g}, 1.33 \mathrm{mmol})$ were added. The resulting solution was stirred at room temperature for 7 days and was then filtered and concentrated in vacuo. Flash chromatography on silica using 15:85 $\rightarrow 1: 2$ ethyl acetate:hexanes $\left(R_{f}=0.53\right.$ in $1: 2$ ethyl acetate:hexanes) afforded the title compound $(0.209 \mathrm{~g}$, $69 \%$ over two steps) as a white solid and as a 1:1 mixture of isomers. IR (neat, $\mathrm{cm}^{-1}$ ) 3218 , $3027,1598,1494,1454,1397,1334,1166,1092,1031,889,813,728,703,669 ; \delta_{\mathrm{H}}(400 \mathrm{MHz}$, $\left.\mathrm{CDCl}_{3}\right) 7.99$ (bs, 1H), $7.81(\mathrm{~d}, 1 \mathrm{H}, J=8.6 \mathrm{~Hz}), 7.63(\mathrm{~d}, 1 \mathrm{H}, J=8.2 \mathrm{~Hz}), 7.32-7.14(\mathrm{~m}, 5 \mathrm{H}), 6.92$ $(\mathrm{m}, 2 \mathrm{H}), 5.49(\mathrm{~m}, 0.5 \mathrm{H}), 5.46(\mathrm{~m}, 0.5 \mathrm{H}), 5.37(\mathrm{~m}, 1 \mathrm{H}), 3.36(\mathrm{~s}, 1 \mathrm{H}), 3.30(\mathrm{AB}, 0.5 \mathrm{H}, J=16.4$ $\mathrm{Hz}), 3.27(\mathrm{AB}, 0.5 \mathrm{H}, J=16.4 \mathrm{~Hz}), 2.46(\mathrm{~s}, 1.5 \mathrm{H}), 2.45(\mathrm{~s}, 1.5 \mathrm{H}), 2.08(\mathrm{~m}, 0.5 \mathrm{H}), 1.61(\mathrm{~m}, 0.5 \mathrm{H})$, 1.60 (apparent tt, $0.5 \mathrm{H}, J=9.4,2.3 \mathrm{~Hz}$ ), 1.52 (apparent tt, $0.5 \mathrm{H}, J=9.0,2.0 \mathrm{~Hz}), 1.35(\mathrm{~m}, 0.5 \mathrm{H})$, $1.14(\mathrm{~m}, 0.5 \mathrm{H}) ; \delta_{\mathrm{C}}\left(100 \mathrm{MHz}, \mathrm{CDCl}_{3}\right) 157.7,156.8,144.2,144.1,136.8,135.5,135.4,133.7$, 131.4, 129.7, 129.6, 129.5, 128.9, 128.4, 128.3, 128.2, 127.9, 127.5, 126.7, 106.9, 106.1, 40.6, 32.4, 22.9, 21.8, 14.8, 10.6, 9.6; $\mathrm{C}_{19} \mathrm{H}_{21} \mathrm{~N}_{2} \mathrm{O}_{2} \mathrm{~S}\left(\mathrm{MH}^{+}\right)$(ESI) Requires: 341.1324 Found: 341.1324 .

\section{1-(2-Methylene-cyclopropyl)-pentan-1-one-tosyl-hydrazone}

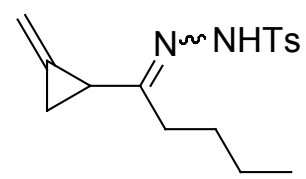

To a solution of 2-methylene-cyclopropanecarboxylic acid methoxy-methyl-amide ( $0.200 \mathrm{~g}, 1.42$ $\mathrm{mmol})$ in diethyl ether $(7.7 \mathrm{~mL})$ at $-78{ }^{\circ} \mathrm{C}$ was added a solution of $\mathrm{BuLi}$ in hexanes $(1.6 \mathrm{M}, 0.98$ $\mathrm{mL}, 1.57 \mathrm{mmol})$. The resulting solution was stirred to room temperature for 4 hours until the reaction was complete. To this solution was added a $1 \mathrm{~N} \mathrm{HCl}$ solution $(10 \mathrm{~mL})$. The reaction solution was extracted with diethyl ether $(4 \times 50 \mathrm{~mL})$ and the combined organic layers were washed with brine, dried over $\mathrm{Na}_{2} \mathrm{SO}_{4}$, filtered and concentrated in vacuo. The crude product was then taken up in dichloromethane $(7.1 \mathrm{~mL}, 0.2 \mathrm{M})$ and $\mathrm{Na}_{2} \mathrm{SO}_{4}$ and $p$-tosylhydrazine $(0.397$ $\mathrm{g}, 2.13 \mathrm{mmol}$ ) were added. The resulting solution was stirred at room temperature for 4 days and was then filtered and concentrated in vacuo. Flash chromatography on silica using 1:2 ethyl acetate:hexanes $\left(R_{f}=0.38\right)$ afforded the title compound $(0.364 \mathrm{~g}, 88 \%$ over two steps $)$ as a white solid and as a 1.1:1 mixture of isomers. IR (neat, $\mathrm{cm}^{-1}$ ) 3116, 2971, 1598, 1455, 1392, 1330, 
$1165,1093,1019,894,812,666 ; \delta_{\mathrm{H}}\left(400 \mathrm{MHz}, \mathrm{CDCl}_{3}\right) 7.89(\mathrm{bs}, 0.54 \mathrm{H}), 7.85 \& 7.83(2 \times \mathrm{d}, 2 \mathrm{H}$, $2 \times J=8.2 \mathrm{~Hz}), 7.52(\mathrm{bs}, 0.46 \mathrm{H}), 7.31(\mathrm{~d}, 2 \mathrm{H}, J=7.8 \mathrm{~Hz}), 5.52(\mathrm{~m}, 0.54 \mathrm{H}), 5.50(\mathrm{~m}, 0.54 \mathrm{H}), 5.45$ $(\mathrm{s}, 0.46 \mathrm{H}), 5.39(\mathrm{~s}, 0.46 \mathrm{H}), 2.43(\mathrm{~s}, 3 \mathrm{H}), 2.27 \& 2.14(\mathrm{~m}, 1 \mathrm{H}), 2.05-1.80(\mathrm{~m}, 2 \mathrm{H}), 1.68$ (apparent tt, $0.54 \mathrm{H}, J=9.0,2.3 \mathrm{~Hz}), 1.49-1.10(\mathrm{~m}, 5.46 \mathrm{H}), 0.87-0.80(\mathrm{~m}, 3 \mathrm{H}) ; \delta_{\mathrm{C}}\left(100 \mathrm{MHz}, \mathrm{CDCl}_{3}\right)$ $160.4,158.7,144.1,135.7,135.5,132.1,129.6,129.6,128.3,106.3,105.2,33.5,28.5,27.9,27.2$, 23.0, 22.3, 22.0, 21.8, 21.8, 14.8, 14.1, 13.9, 10.1, 9.7; $\mathrm{C}_{16} \mathrm{H}_{23} \mathrm{~N}_{2} \mathrm{O}_{2} \mathrm{~S}\left(\mathrm{MH}^{+}\right)$(ESI) Requires: 307.1474 Found: 307.1479.

\section{1-(2-Methylene-cyclopropyl)-but-3-en-1-one-tosyl-hydrazone}

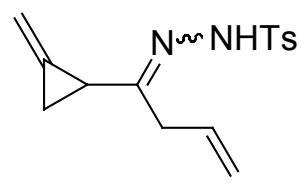

To a solution of 2-methylene-cyclopropanecarboxylic acid methoxy-methyl-amide $(0.200 \mathrm{~g}, 1.42$ $\mathrm{mmol})$ in THF $(7 \mathrm{~mL})$ at $0{ }^{\circ} \mathrm{C}$ was added a solution of allylMgBr in diethyl ether $(1.0 \mathrm{M}, 4.26$ $\mathrm{mL}, 4.26 \mathrm{mmol})$. The resulting solution was then stirred to room temperature for 12 hours until the reaction was complete. To this solution was added a $1 \mathrm{~N} \mathrm{HCl}$ solution $(10 \mathrm{~mL})$. The reaction solution was extracted with diethyl ether $(4 \times 50 \mathrm{~mL})$ and the combined organic layers were washed with brine, dried over $\mathrm{Na}_{2} \mathrm{SO}_{4}$, filtered and concentrated in vacuo. The crude product was then taken up in absolute ethanol $(10 \mathrm{~mL}, 0.14 \mathrm{M})$ and molecular sieves and $p$ tosylhydrazine $(0.397 \mathrm{~g}, 2.13 \mathrm{mmol})$ were added. The resulting solution was stirred at room temperature for 12 hours and was then filtered and concentrated in vacuo. Flash chromatography on silica using 1:3 ethyl acetate:hexanes $\left(R_{f}=0.21\right)$ afforded the title compound $(0.150 \mathrm{~g}, 36 \%$ over two steps) as a light yellow solid and as a 1:1 mixture of isomers. IR (neat, $\mathrm{cm}^{-1}$ ) 3610 , 3215, 2921, 1391, 1330, 1163; $\delta_{\mathrm{H}}\left(400 \mathrm{MHz}, \mathrm{CDCl}_{3}\right) 8.21(\mathrm{~s}, 0.5 \mathrm{H}), 7.85(\mathrm{~d}, 1 \mathrm{H}, J=6.7 \mathrm{~Hz})$, $7.81(\mathrm{~d}, 1 \mathrm{H}, J=6.7 \mathrm{~Hz}), 7.65(\mathrm{~s}, 0.5 \mathrm{H}), 7.31(\mathrm{~d}, 1 \mathrm{H}, J=3.9 \mathrm{~Hz}), 7.29(\mathrm{~d}, 1 \mathrm{H}, J=3.8 \mathrm{~Hz}), 5.67$ $(\mathrm{m}, 1 \mathrm{H}), 5.51(\mathrm{~m}, 1 \mathrm{H}), 5.44(\mathrm{~m}, 1 \mathrm{H}), 5.02(\mathrm{~m}, 1 \mathrm{H}), 4.97(\mathrm{dd}, 0.5 \mathrm{H}, J=17.1,1.6 \mathrm{~Hz}, \mathrm{~Hz}), 4.79$ $(\mathrm{dd}, 0.5 \mathrm{H}, J=17.4,1.2 \mathrm{~Hz}), 2.76(\mathrm{~m}, 0.5 \mathrm{H}), 2.68(\mathrm{~m}, 0.5 \mathrm{H}), 2.43(\mathrm{~s}, 1.5 \mathrm{H}), 2.42(\mathrm{~s}, 1.5 \mathrm{H}), 2.38$ $(\mathrm{m}, 0.5 \mathrm{H}), 2.21(\mathrm{~m}, 0.5 \mathrm{H}), 1.69(\mathrm{~m}, 0.5 \mathrm{H}), 1.49(\mathrm{~m}, 0.5 \mathrm{H}), 1.34(\mathrm{~m}, 0.5 \mathrm{H}), 1.26(\mathrm{~m}, 1 \mathrm{H}) ; \delta_{\mathrm{C}}(100$ $\left.\mathrm{MHz}, \mathrm{CDCl}_{3}\right)$ 157.2, 157.1, 144.19, 144.15, 135.6,135.5,133.6, 131.4, 130.4, 129.8, 129.68, 129.67, 128.3 , 128.2, 117.8, 117.3, 106.6, 106.1, 38.1, 30.6, 22.5, 21.84, 21.82, 15.0, 10.5, 9.4 ; $\mathrm{C}_{15} \mathrm{H}_{19} \mathrm{~N}_{2} \mathrm{O}_{2} \mathrm{~S}\left(\mathrm{MH}^{+}\right)(\mathrm{ESI})$ Requires: 291.1161 Found: 291.1162.

\section{4-(tert-Butyl-dimethyl-silanyloxy)-1-(2-methylene-cyclopropyl)- butan-1-one-tosyl-hydrazone}

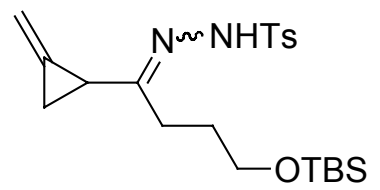

To a solution of 2-methylene-cyclopropanecarboxylic acid methoxy-methyl-amide $(0.249 \mathrm{~g}, 1.76$ $\mathrm{mmol})$ in THF $(9.5 \mathrm{~mL})$ at $-78^{\circ} \mathrm{C}$ was added a solution of the requisite Grignard reagent in THF $(0.40 \mathrm{M}, 8.7 \mathrm{~mL}, 3.48 \mathrm{mmol})$. The resulting solution was then stirred to room temperature for 10 hours. To this solution was added a $1 \mathrm{~N} \mathrm{HCl}$ solution $(10 \mathrm{~mL})$. The reaction solution was then extracted with diethyl ether $(4 \times 50 \mathrm{~mL})$ and the combined organic layers were washed with brine, dried over $\mathrm{Na}_{2} \mathrm{SO}_{4}$, filtered and concentrated in vacuo. The crude product was then purified on silica using 1:9 diethyl ether:hexanes and the resulting ketone was taken up in dichloromethane $(8.8 \mathrm{~mL}, 0.2 \mathrm{M})$ and $\mathrm{Na}_{2} \mathrm{SO}_{4}$ and $p$-tosylhydrazine $(0.650 \mathrm{~g}, 3.49 \mathrm{mmol})$ were added. The 
resulting solution was stirred at room temperature for 4.5 days and was then filtered and concentrated in vacuo. Flash chromatography on silica using 1:4 diethyl ether:hexanes $\left(R_{f}=\right.$ 0.25-0.14) afforded the title compound (0.741 g, 99\% over two steps) a clear oil as a 1.2:1 mixture of isomers that upon solidifying under high vacuum, was isolated as a white solid and as a 11:1 mixture of isomers. ${ }^{4}$ IR (neat, $\mathrm{cm}^{-1}$ ) 3216, 2931, 2956, 2739, 2255, 1915, 1799, 1747 , $1632,1599,1495,1469,1392,1336,1257,1168,1093,1020,897,836,812,777,706,665 ; \delta_{\mathrm{H}}$ $\left(400 \mathrm{MHz}, \mathrm{CDCl}_{3}\right) 8.78(\mathrm{~s}, 0.55 \mathrm{H}), 8.11(\mathrm{bs}, 0.45 \mathrm{H}), 7.79(\mathrm{~m}, 2 \mathrm{H}), 7.24(\mathrm{~m}, 2 \mathrm{H}), 5.46(\mathrm{~s}$, $0.88 \mathrm{H}), 5.38(\mathrm{~s}, 0.56 \mathrm{H}), 5.30(\mathrm{~s}, 0.56 \mathrm{H}), 3.44(\mathrm{bs}, 0.91 \mathrm{H}), 3.29(\mathrm{~m}, 1.09 \mathrm{H}), 2.37(\mathrm{~s}, 3 \mathrm{H}), 2.24-$ $1.91(\mathrm{~m}, 3 \mathrm{H}), 1.66-1.50(\mathrm{~m}, 2.45 \mathrm{H}), 1.39(\mathrm{bs}, 1 \mathrm{H}), 1.21(\mathrm{~m}, 0.55 \mathrm{H}), 0.90(\mathrm{~s}, 4.85 \mathrm{H}), 0.81(\mathrm{~s}$, $4.15 \mathrm{H}), 0.08(\mathrm{~s}, 3.20 \mathrm{H}),-0.06(\mathrm{~s}, 2.8 \mathrm{H}) ; \delta_{\mathrm{C}}\left(100 \mathrm{MHz}, \mathrm{CDCl}_{3}\right) 158.5,157.7,143.9,143.5,136.1$, 135.6, 132.4, 129.8, 129.5, 129.3, 128.2, 128.0, 106.1, 104.9, 62.2, 60.8, 29.5, 29.1, 28.9, 26.1, 26.0, 23.1, 22.0, 21.7, 18.5, 18.4, 15.0, 10.2, 9.5, -5.0, -5.3; $\mathrm{C}_{21} \mathrm{H}_{35} \mathrm{~N}_{2} \mathrm{O}_{3} \mathrm{SSi}\left(\mathrm{MH}^{+}\right)$(ESI) Requires: 423.2132 Found: 423.2132.

\section{[1,3]-Dithian-2-yl-(2-methylene-cyclopropyl)-methanone-tosyl-hydrazone}

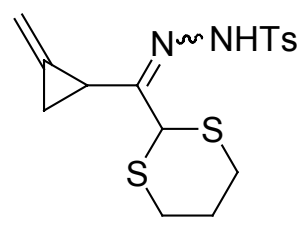

The Grignard solution was prepared by the dropwise addition of BuLi in hexanes (1.6 M, 1.8 $\mathrm{mL}, 2.88 \mathrm{mmol})$ to a solution of 1,3-dithiane $(0.340 \mathrm{~g}, 2.83 \mathrm{mmol})$ in THF $(30 \mathrm{~mL})$ at $-78{ }^{\circ} \mathrm{C}$. The solution was stirred to $-40{ }^{\circ} \mathrm{C}$ for 1 hour before being cooled back down to $-78{ }^{\circ} \mathrm{C}$ prior to the addition of 2-methylene-cyclopropanecarboxylic acid methoxy-methyl-amide $(0.184 \mathrm{~g}, 1.30$ $\mathrm{mmol})$ in THF $(8 \mathrm{~mL})$. The resulting solution was then stirred to room temperature overnight. The reaction solution was quenched by the addition of water $(20 \mathrm{~mL})$, followed by extraction with diethyl ether $(4 \times 50 \mathrm{~mL})$. The combined organic layers were then washed with brine, dried over $\mathrm{Na}_{2} \mathrm{SO}_{4}$, filtered and concentrated in vacuo. The crude product was purified on silica from excess 1,3-dithiane using 1:4 diethyl ether:hexanes to afford the ketone product which was subsequently taken up in dichloromethane $(14.4 \mathrm{~mL}, 0.2 \mathrm{M})$ and $\mathrm{Na}_{2} \mathrm{SO}_{4}$ and $p$-tosylhydrazine $(0.484 \mathrm{~g}, 2.60 \mathrm{mmol})$ were added. $p$-Toluene sulfonic acid monohydrate $(0.0247 \mathrm{~g}, 10 \mathrm{~mol} \%)$ was then added in order to the reaction to proceed. The resulting solution was stirred at room temperature for 8 days and was then filtered and concentrated in vacuo. Flash chromatography on silica using 15:85 ethyl acetate:hexanes $\left(R_{f}=0.13\right)$ afforded the title compound $(0.150 \mathrm{~g}, 31 \%$ over two steps) as a white solid and as a single isomer, $\mathrm{mp}=134-137{ }^{\circ} \mathrm{C}\left(\mathrm{CH}_{2} \mathrm{Cl}_{2}\right)$. IR (neat, $\mathrm{cm}^{-}$ 1) $3214,2927,1397,1335,1159 ; \delta_{\mathrm{H}}\left(400 \mathrm{MHz}, \mathrm{CDCl}_{3}\right) 8.40(\mathrm{~s}, 1 \mathrm{H}), 7.89(\mathrm{~d}, 2 \mathrm{H}, J=8.2 \mathrm{~Hz})$, $7.32(\mathrm{~d}, 2 \mathrm{H}, J=8.1 \mathrm{~Hz}), 5.66(\mathrm{~m}, 1 \mathrm{H}), 5.57(\mathrm{~m}, 1 \mathrm{H}), 4.16(\mathrm{~s}, 1 \mathrm{H}), 3.09(\mathrm{~m}, 1 \mathrm{H}), 2.96(\mathrm{~m}, 1 \mathrm{H})$, $2.58(\mathrm{~m}, 2 \mathrm{H}), 2.42(\mathrm{~s}, 3 \mathrm{H}), 2.19(\mathrm{~m}, 1 \mathrm{H}), 1.95(\mathrm{~m}, 2 \mathrm{H}), 1.79(\mathrm{~m}, 1 \mathrm{H}), 1.26(\mathrm{~m}, 1 \mathrm{H}) ; \delta_{\mathrm{C}}(100$ $\mathrm{MHz}_{\mathrm{CDCl}}$ ) 154.2, 144.4, 135.4, 129.8, 129.0, 128.3, 107.3, 45.0, 27.8, 25.7, 21.9, 13.9, 11.1; $\mathrm{C}_{16} \mathrm{H}_{21} \mathrm{~N}_{2} \mathrm{O}_{2} \mathrm{~S}_{3}\left(\mathrm{M}^{+}\right)$(ESI) Requires: 369.0759 Found: 369.0751. 


\section{(2-Methylene-cyclopropyl)-phenyl-methanone-tosyl-hydrazone}

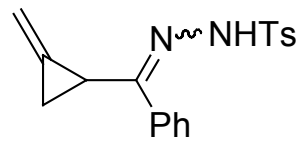

To a solution of 2-methylene-cyclopropanecarboxylic acid methoxy-methyl-amide $(0.210 \mathrm{~g}, 1.48$ mmol $)$ in diethyl ether $(8.1 \mathrm{~mL})$ at $-78{ }^{\circ} \mathrm{C}$ was added a solution of $\mathrm{PhMgBr}$ in diethyl ether $(2.5$ $\mathrm{M}, 1.3 \mathrm{~mL}, 3.25 \mathrm{mmol}$ ). The resulting solution was then stirred to room temperature overnight. To this solution was added a $1 \mathrm{~N} \mathrm{HCl}$ solution $(10 \mathrm{~mL})$. The reaction solution was then extracted with diethyl ether $(4 \times 50 \mathrm{~mL})$ and the combined organic layers were washed with brine, dried over $\mathrm{Na}_{2} \mathrm{SO}_{4}$, filtered and concentrated in vacuo. The crude product was then taken up in dichloromethane $(7.4 \mathrm{~mL}, 0.2 \mathrm{M})$ and $\mathrm{Na}_{2} \mathrm{SO}_{4}, p$-tosylhydrazine $(0.551 \mathrm{~g}, 2.96 \mathrm{mmol})$ and $p$ toluene sulfonic acid monohydrate $(0.0282 \mathrm{~g}, 10 \mathrm{~mol} \%)$ were added. The resulting solution was stirred at room temperature for 6 days and was then filtered and concentrated in vacuo. Flash chromatography on silica using 15:85 ethyl acetate:hexanes $\left(R_{f}=0.21\right)$ afforded the title compound ( $0.357 \mathrm{~g}, 74 \%$ over two steps) as a white solid and as a 2.3:1 mixture of isomers. IR $\left(\right.$ neat, $\left.\mathrm{cm}^{-1}\right)$ 3213, 2926, 1598, 1440, 1383, 1323, 1344, 1305, 1167, 1089, 1015, 895, 814, 768, $666 ; \delta_{\mathrm{H}}\left(400 \mathrm{MHz}, \mathrm{CDCl}_{3}\right) 8.41(\mathrm{bs}, 0.60 \mathrm{H}), 7.88(\mathrm{~d}, 1.2 \mathrm{H}, J=8.2 \mathrm{~Hz}), 7.78(\mathrm{~d}, 0.8 \mathrm{H}, J=8.2$ $\mathrm{Hz}), 7.61(\mathrm{dd}, 1.2 \mathrm{H}, J=6.6,3.1 \mathrm{~Hz}), 7.40(\mathrm{~m}, 1.2 \mathrm{H}), 7.33(\mathrm{~m}, 3.8 \mathrm{H}), 7.23(\mathrm{bs}, 0.4 \mathrm{H}), 7.00(\mathrm{dd}$, $0.8 \mathrm{H}, J=6.6,2.7 \mathrm{~Hz}), 5.62(\mathrm{~m}, 0.6 \mathrm{H}), 5.58(\mathrm{~m}, 0.6 \mathrm{H}), 5.46(\mathrm{~m}, 0.4 \mathrm{H}), 5.40(\mathrm{~m}, 0.4 \mathrm{H}), 2.54(\mathrm{~m}$, $0.4 \mathrm{H}), 2.45(\mathrm{~s}, 1.2 \mathrm{H}), 2.41(\mathrm{~s}, 1.8 \mathrm{H}), 2.34$ (dddd, $0.6 \mathrm{H}, J=8.8,6.2,2.5,2.3 \mathrm{~Hz}), 1.84$ (apparent tt, $0.6 \mathrm{H}, J=9.0,2.3 \mathrm{~Hz}), 1.49(\mathrm{~m}, 0.4 \mathrm{H}), 1.21(\mathrm{~m}, 1 \mathrm{H}) ; \delta_{\mathrm{C}}\left(100 \mathrm{MHz}, \mathrm{CDCl}_{3}\right) 157.5,151.8$, $144.4,144.2$, 136.2, 135.7, 131.6, 130.4, 130.1, 129.8, 129.8, 129.7, 129.6, 129.5, 128.7, 128.3, $128.2,128.1,127.7,127.6,125.6,107.2,106.0,22.8,21.9,21.8,13.8,10.6,9.9 ; \mathrm{C}_{18} \mathrm{H}_{19} \mathrm{~N}_{2} \mathrm{O}_{2} \mathrm{~S}$ $\left(\mathrm{MH}^{+}\right)(\mathrm{ESI})$ Requires: 327.1161 Found: 327.1163.

Furan-2-yl-(2-methylene-cyclopropyl)-methanone-tosyl-hydrazone

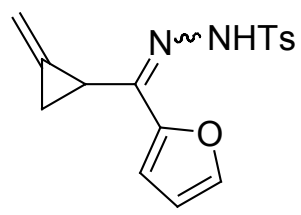

The Grignard solution was prepared by the dropwise addition of BuLi in hexanes (1.6 M, 1.8 $\mathrm{mL}, 2.88 \mathrm{mmol})$ to a solution of furan $(0.200 \mathrm{~g}, 2.94 \mathrm{mmol})$ in THF $(31 \mathrm{~mL})$ at $-78{ }^{\circ} \mathrm{C}$. The solution was stirred to $-40{ }^{\circ} \mathrm{C}$ for 45 minutes before being cooled back down to $-78{ }^{\circ} \mathrm{C}$ prior to the addition of 2-methylene-cyclopropanecarboxylic acid methoxy-methyl-amide $(0.192 \mathrm{~g}, 1.36$ $\mathrm{mmol})$ in THF $(8 \mathrm{~mL})$. The resulting solution was stirred to room temperature overnight. The reaction solution was quenched by the addition of water $(20 \mathrm{~mL})$, followed by extraction with diethyl ether $(4 \times 50 \mathrm{~mL})$. The combined organic layers were then washed with brine, dried over $\mathrm{Na}_{2} \mathrm{SO}_{4}$, filtered and concentrated in vacuo. The crude product was purified on silica using 1:4 diethyl ether:hexanes to afford the ketone product which was subsequently taken up in dichloromethane $(14.4 \mathrm{~mL}, 0.2 \mathrm{M})$ and $\mathrm{Na}_{2} \mathrm{SO}_{4}, p$-tosylhydrazine $(0.505 \mathrm{~g}, 2.71 \mathrm{mmol})$, and $p$ toluene sulfonic acid monohydrate $(0.0257 \mathrm{~g}, 10 \mathrm{~mol} \%)$ were added. The resulting solution was stirred at room temperature for 8 days and was then filtered and concentrated in vacuo. Flash chromatography on silica using 15:85 ethyl acetate:hexanes $\left(R_{f}=0.11\right)$ afforded the title compound $(0.190 \mathrm{~g}, 44 \%$ over two steps) as a white solid and as a 2.1:1 mixture of isomers. IR (neat, $\left.\mathrm{cm}^{-1}\right) 3613,2927,2855,1746,1518,1488,1162 ; \delta_{\mathrm{H}}\left(400 \mathrm{MHz}, \mathrm{CDCl}_{3}\right) 9.36(\mathrm{~s}, 0.68 \mathrm{H})$, 
$8.57(\mathrm{~s}, 0.32 \mathrm{H}), 7.87(\mathrm{~d}, 0.64 \mathrm{H}, J=8.4 \mathrm{~Hz}), 7.81(\mathrm{~d}, 1.36 \mathrm{H}, J=8.4 \mathrm{~Hz}), 7.56(\mathrm{~d}, 0.68 \mathrm{H}, J=1.8$ $\mathrm{Hz}), 7.39(\mathrm{~d}, 0.32 \mathrm{H}, J=1.8 \mathrm{~Hz}), 7.27(\mathrm{~m}, 2 \mathrm{H}), 6.82(\mathrm{~d}, 0.68 \mathrm{H}, J=3.7 \mathrm{~Hz}), 6.70(\mathrm{~d}, 0.32 \mathrm{H}, J=$ $3.4 \mathrm{~Hz}), 6.50(\mathrm{dd}, 0.68 \mathrm{H}, J=3.7,1.9 \mathrm{~Hz}), 6.35(\mathrm{dd}, 0.32 \mathrm{H}, J=3.5,1.8 \mathrm{~Hz}), 5.64(\mathrm{~m}, 0.64 \mathrm{H})$, $5.39(\mathrm{~m}, 1 \mathrm{H}, 1.36 \mathrm{H}), 2.42(\mathrm{~m}, 0.68 \mathrm{H}), 2.38(\mathrm{~s}, 2.04 \mathrm{H}), 2.37(\mathrm{~s}, 0.96 \mathrm{H}), 2.28(\mathrm{~m}, 0.32 \mathrm{H}), 1.82(\mathrm{~m}$, $0.32 \mathrm{H}), 1.63(\mathrm{~m}, 0.68 \mathrm{H}), 1.53(\mathrm{~m}, 0.68 \mathrm{H}), 1.41(\mathrm{~m}, 0.32 \mathrm{H}) ; \delta_{\mathrm{C}}\left(100 \mathrm{MHz}, \mathrm{CDCl}_{3}\right)$ 150.6, 149.0, 144.4, 144.2, 143.9, 143.8, 139.3, 135.6, 132.4, 129.8, 129.6, 128.2, 114.8, 111.9, 111.8, 111.6, 107.4, 104.1, 21.8, 18.9, 13.2, 10.7, $9.8 ; \mathrm{C}_{16} \mathrm{H}_{17} \mathrm{~N}_{2} \mathrm{O}_{3} \mathrm{~S}\left(\mathrm{MH}^{+}\right)$(ESI) Requires: 317.0954 Found: 317.0960 .

\section{1-(2-Methylene-cyclopropyl)-ethanone oxime}

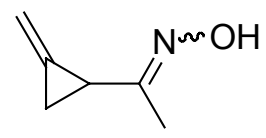

To a solution of 2-methylene-cyclopropanecarboxylic acid methoxy-methyl-amide $(0.565 \mathrm{~g}, 4.00$ $\mathrm{mmol})$ in THF $(23.7 \mathrm{~mL})$ at $-78^{\circ} \mathrm{C}$ was added a solution of $\mathrm{MeMgBr}$ in diethyl ether (3.2 M, 2.0 $\mathrm{mL}, 6.40 \mathrm{mmol})$. The resulting white suspension was stirred to room temperature for 4 hours until the reaction was complete. To this solution was added a $1 \mathrm{~N} \mathrm{HCl}$ solution $(10 \mathrm{~mL})$ and the reaction solution was extracted with diethyl ether $(4 \times 50 \mathrm{~mL})$. The combined organic layers were then washed with brine, dried over $\mathrm{Na}_{2} \mathrm{SO}_{4}$, filtered and concentrated in vacuo to afford a crude product that was taken up in a water $(3.3 \mathrm{~mL}) /$ ethanol $(14.3 \mathrm{~mL})$ solution containing sodium acetate $(3.37 \mathrm{~g}, 41.1 \mathrm{mmol})$ and hydroxylamine hydrochloride $(1.02 \mathrm{~g}, 14.7 \mathrm{mmol})$. The resulting solution was stirred at room temperature overnight until complete by TLC. Brine was added and the resulting solution was extracted with dichloromethane $(4 \times 50 \mathrm{~mL})$. The combined organic layers were then dried with $\mathrm{Na}_{2} \mathrm{SO}_{4}$, filtered and concentrated in vacuo. Flash chromatography on silica using 1:3 diethyl ether:hexanes $\left(R_{f}=0.25-0.22\right)$ afforded the title compound $(0.444 \mathrm{~g}, 100 \%)$ as a clear oil and as a 2.8:1 mixture of isomers. IR (neat, $\left.\mathrm{cm}^{-1}\right) 3241$, 2922, 1652, 1433, 1370, 1316, 1273, 1116, 1035, 989, 955, 896; $\delta_{\mathrm{H}}\left(400 \mathrm{MHz}, \mathrm{CDCl}_{3}\right) 9.39(\mathrm{bs}$, $1 \mathrm{H}), 5.56(\mathrm{~m}, 0.74 \mathrm{H}), 5.53(\mathrm{~m}, 1 \mathrm{H}), 5.50(\mathrm{~m}, 0.26 \mathrm{H}), 3.17(\mathrm{~m}, 0.26 \mathrm{H}), 2.34(\mathrm{~m}, 0.74 \mathrm{H}), 1.69(\mathrm{~s}$, $2.22 \mathrm{H}), 1.67$ (apparent tt, $0.74 \mathrm{H}, J=9.4,2.3 \mathrm{~Hz}), 1.59(\mathrm{~s}, 0.78 \mathrm{H}), 1.54$ (apparent tt, $0.26 \mathrm{H}, J=$ 9.2, $2.1 \mathrm{~Hz}), 1.45(\mathrm{~m}, 0.26 \mathrm{H}), 1.35(\mathrm{~m}, 0.74 \mathrm{H}) ; \delta_{\mathrm{C}}\left(125 \mathrm{MHz}, \mathrm{CDCl}_{3}\right)$ 158.0, 157.2, 131.8, 131.4, 106.1, 105.5, 20.3, 14.9, 14.3, 10.2, 9.8, 9.4; $\mathrm{C}_{6} \mathrm{H}_{8} \mathrm{NO}\left(\mathrm{M}-\mathrm{H}^{+}\right)(\mathrm{EI})$ Requires: 94.0657 Found: 94.0660.

\section{General Procedure for the Dienamine}

To a sealed oven-dried vial purged with argon, was added the MCP hydrazone followed by DME $(0.01 \mathrm{M})$ and TMEDA (redistilled, 1 equivalent) under a cone of argon with stirring. $\mathrm{MgCl}_{2}(10$ mol\%) was then added and the vial was immediately sealed and heated at $120{ }^{\circ} \mathrm{C}$ until the reaction was judged complete by TLC. The resulting solution was evaporated and loaded directly onto the silica column and purified by flash chromatography to afford the title compound in the indicated yield. 
3,5-Dimethyl-1-(toluene-4-sulfonyl)-1,2-dihydro-pyridazine

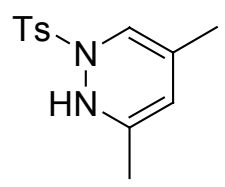

According to the general procedure outlined above using 1-(2-methylene-cyclopropyl)-ethanonetosyl-hydrazone $(0.0200 \mathrm{~g}, 0.0757 \mathrm{mmol})$, TMEDA (11.3 $\mu \mathrm{L}, 0.0754 \mathrm{mmol})$ and $\mathrm{MgCl}_{2}(0.7 \mathrm{mg}$, $10 \mathrm{~mol} \%$ ) in DME $(7.5 \mathrm{~mL})$ at $120{ }^{\circ} \mathrm{C}$ for 3.5 hours, the title compound could be obtained after flash chromatography on silica using 1:4 ethyl acetate:hexanes $\left(\mathrm{R}_{\mathrm{f}}=0.26\right.$ in $25 \%$ ethyl acetate in hexanes) in $85 \%$ yield $(0.0170 \mathrm{~g})$ and with a selectivity of $>20: 1$ over the azadiene product. White solid. IR (neat, $\mathrm{cm}^{-1}$ ) 3240, 2925, 1597, 1441, 1347, 1186, 1166, 1091, 813, 716, 666; $\delta_{\mathrm{H}}$ $\left(500 \mathrm{MHz}, \mathrm{CDCl}_{3}\right) 7.65$ (d, 2H, $\left.J=8.3 \mathrm{~Hz}\right), 7.32(\mathrm{~d}, 2 \mathrm{H}, J=8.1 \mathrm{~Hz}), 7.01$ (bs, $\left.1 \mathrm{H}\right), 5.99(\mathrm{~s}, 1 \mathrm{H})$, $5.60(\mathrm{~s}, 1 \mathrm{H}), 2.46(\mathrm{~s}, 3 \mathrm{H}), 1.92(\mathrm{~s}, 3 \mathrm{H}), 1.88(\mathrm{~s}, 3 \mathrm{H}) ; \delta_{\mathrm{C}}\left(125 \mathrm{MHz}, \mathrm{CDCl}_{3}\right)$ 145.2, 134.6, 130.5, 130.0, 128.7, 118.0, 117.6, 107.5, 21.9, 12.1, 10.9; $\mathrm{C}_{13} \mathrm{H}_{17} \mathrm{~N}_{2} \mathrm{O}_{2} \mathrm{~S}\left(\mathrm{MH}^{+}\right)$(ESI) Requires: 265.1005 Found: 265.0998.

\section{3-Ethyl-5-methyl-1-(toluene-4-sulfonyl)-1,2-dihydro-pyridazine}

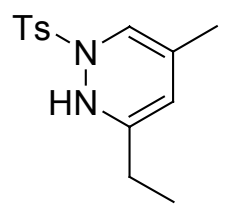

According to the general procedure outlined above using 1-(2-methylene-cyclopropyl)-propan-1one-tosyl-hydrazone $(0.0150 \mathrm{~g}, 0.0539 \mathrm{mmol})$, TMEDA $(8.1 \mu \mathrm{L}, 0.0540 \mathrm{mmol})$ and $\mathrm{MgCl}_{2}(0.5$ $\mathrm{mg}, 10 \mathrm{~mol} \%)$ in DME $(5.4 \mathrm{~mL})$ at $120{ }^{\circ} \mathrm{C}$ for 4 hours, the title compound could be obtained after flash chromatography on silica using 16:84 diethyl ether:hexanes $\left(\mathrm{R}_{f}=0.23\right.$ in ethyl acetate/hexanes $1: 3)$ in $77 \%$ combined yield $(0.0110 \mathrm{~g}$ of dienamine $)$ and with a selectivity of $>$ 20:1 over the azadiene product. Colorless oil. IR (film, $\mathrm{cm}^{-1}$ ) 3229, 2963, 2927, 1593, 1440, $1344 ; \delta_{\mathrm{H}}\left(400 \mathrm{MHz}, \mathrm{CDCl}_{3}\right) 7.67(\mathrm{~d}, 2 \mathrm{H}, J=8.2 \mathrm{~Hz}), 7.31(\mathrm{~d}, 2 \mathrm{H}, J=8.6 \mathrm{~Hz}), 6.89(\mathrm{~s}, 1 \mathrm{H}), 5.95$ $(\mathrm{s}, 1 \mathrm{H}), 5.63(\mathrm{~s}, 1 \mathrm{H}), 2.46(\mathrm{~s}, 3 \mathrm{H}), 2.27(\mathrm{q}, 2 \mathrm{H}, J=7.41 \mathrm{~Hz}), 1.93$, (s, 3H), $1.06(\mathrm{t}, 3 \mathrm{H}, J=7.41$ $\mathrm{Hz}) ; \delta_{\mathrm{C}}\left(100 \mathrm{MHz}, \mathrm{CDCl}_{3}\right) 145.2,136.9,134.6,130.0,128.7,117.9,117.7,105.8,21.9,18.3$, 12.7, 12.2; $\mathrm{C}_{14} \mathrm{H}_{18} \mathrm{~N}_{2} \mathrm{O}_{2} \mathrm{~S}\left(\mathrm{M}^{+}\right)$(EI) Requires: 278.1089 Found: 278.1083.

\section{3-Benzyl-5-methyl-1-(toluene-4-sulfonyl)-1,2-dihydro-pyridazine}

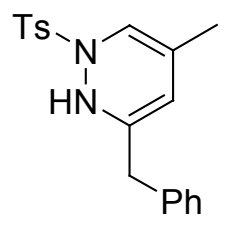

According to the general procedure outlined above using 1-(2-methylene-cyclopropyl)-2-phenylethanone-tosyl-hydrazone $(0.0150 \mathrm{~g}, 0.0441 \mathrm{mmol})$, TMEDA ( $7.1 \mu \mathrm{L}, 0.0474 \mathrm{mmol})$ and $\mathrm{MgCl}_{2}$ $(0.4 \mathrm{mg}, 10 \mathrm{~mol} \%)$ in DME $(4.5 \mathrm{~mL})$ at $120^{\circ} \mathrm{C}$ for 4 hours, the title compound could be obtained after flash chromatography on silica using 16:84 diethyl ether:hexanes $\left(\mathrm{R}_{f}=0.32\right.$ in 1:3 ethyl acetate:hexanes) in $70 \%$ combined yield $(0.0095 \mathrm{~g}$ of dienamine) and with a selectivity of 10:1 over the azadiene product. Colorless oil. IR (neat, $\mathrm{cm}^{-1}$ ) 3242, 2921, 1494, 1337, 1164, 1089; $\delta_{\mathrm{H}}$ $\left(400 \mathrm{MHz}, \mathrm{CDCl}_{3}\right) 7.67(\mathrm{~d}, 2 \mathrm{H}, J=8.22 \mathrm{~Hz}), 7.31(\mathrm{~d}, 2 \mathrm{H}, J=8.0 \mathrm{~Hz}), 7.24(\mathrm{~m}, 2 \mathrm{H}), 7.17(\mathrm{~m}$, $1 \mathrm{H}), 7.03(\mathrm{~d}, 2 \mathrm{H}, J=7.24 \mathrm{~Hz}), 6.80(\mathrm{~s}, 1 \mathrm{H}), 6.03(\mathrm{bs}, 1 \mathrm{H}), 5.54(\mathrm{~s}, 1 \mathrm{H}), 3.52(\mathrm{~s}, 2 \mathrm{H}), 2.46(\mathrm{~s}$, 
$3 \mathrm{H}), 1.91(\mathrm{~s}, 3 \mathrm{H}) ; \delta_{\mathrm{C}}\left(100 \mathrm{MHz}, \mathrm{CDCl}_{3}\right) 145.3,138.9,134.7,133.2,130.1,128.9,128.7,128.6$, 126.5, 118.8, 117.6, 108.6, 31.7, 21.9, 12.2; $\mathrm{C}_{19} \mathrm{H}_{20} \mathrm{~N}_{2} \mathrm{O}_{2} \mathrm{~S}\left(\mathrm{M}^{+}\right)$(EI) Requires: 340.1246 Found: 340.1237.

Additionally, the reaction could be carried out under higher concentration $(0.028 \mathrm{M})$ using 1-(2methylene-cyclopropyl)-2-phenyl-ethanone-tosyl-hydrazone (0.0512 g, $0.150 \mathrm{mmol})$, TMEDA $(23 \mu \mathrm{L}, 0.153 \mathrm{mmol})$ and $\mathrm{MgCl}_{2}(1.4 \mathrm{mg}, 10 \mathrm{~mol} \%)$ in DME $(5.4 \mathrm{~mL})$ at $120{ }^{\circ} \mathrm{C}$ for 5 hours. The title compound could be obtained after flash chromatography on silica using 16:84 diethyl ether:hexanes $\left(\mathrm{R}_{f}=0.32\right.$ in $1: 3$ ethyl acetate:hexanes) in $74 \%$ combined yield $(0.0345 \mathrm{~g}$ of dienamine) and with a selectivity of 10:1 over the azadiene product.

\section{3-Butyl-5-methyl-1-(toluene-4-sulfonyl)-1,2-dihydro-pyridazine}

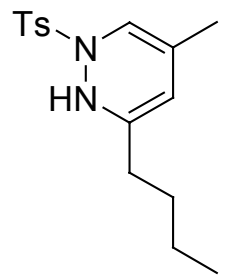

According to the general procedure outlined above using 1-(2-methylene-cyclopropyl)-pentan-1one-tosyl-hydrazone $(0.0150 \mathrm{~g}, 0.0490 \mathrm{mmol})$, TMEDA $(7.4 \mu \mathrm{L}, 0.0494 \mathrm{mmol})$ and $\mathrm{MgCl}_{2}(0.5$ $\mathrm{mg}, 10 \mathrm{~mol} \%)$ in DME $(4.5 \mathrm{~mL})$ at $120{ }^{\circ} \mathrm{C}$ for 4 hours, the title compound could be obtained after flash chromatography on silica using 16:84 diethyl ether:hexanes $\left(\mathrm{R}_{f}=0.34\right.$ in ethyl acetate/hexanes 1:3) in 91\% combined yield (0.0132 g of dienamine) and with a selectivity of $>$ 20:1 over the azadiene product. Colorless oil. IR (neat, $\mathrm{cm}^{-1}$ ) 3611, 2921, 2860, 1701, 1521, 1167; $\delta_{\mathrm{H}}\left(400 \mathrm{MHz}, \mathrm{CDCl}_{3}\right) 7.67(\mathrm{~d}, 2 \mathrm{H}, J=6.63 \mathrm{~Hz}), 7.31(\mathrm{~d}, 2 \mathrm{H}, J=7.8 \mathrm{~Hz}), 6.88(\mathrm{~s}, 1 \mathrm{H})$, $6.00(\mathrm{~s}, 1 \mathrm{H}), 5.61(\mathrm{~s}, 1 \mathrm{H}), 2.45(\mathrm{~s}, 3 \mathrm{H}), 2.18(\mathrm{t}, 2 \mathrm{H}, J=7.4 \mathrm{~Hz}), 1.93(\mathrm{~s}, 3 \mathrm{H}), 1.40(\mathrm{~m}, 2 \mathrm{H}), 1.26$ $(\mathrm{m}, 2 \mathrm{H}), 0.85(\mathrm{t}, 3 \mathrm{H}, J=7.4 \mathrm{~Hz}) ; \delta_{\mathrm{C}}\left(100 \mathrm{MHz}, \mathrm{CDCl}_{3}\right) 145.2,135.4,134.6,130.0,128.7,117.9$, 117.6, 106.3, 30.7, 24.7, 22.7, 21.9, 14.1, 12.2; $\mathrm{C}_{16} \mathrm{H}_{23} \mathrm{~N}_{2} \mathrm{O}_{2} \mathrm{~S}\left(\mathrm{MH}^{+}\right)$(ESI) Requires: 307.1474 Found: 307.1480 .

\section{3-Allyl-5-methyl-1-(toluene-4-sulfonyl)-1,2-dihydro-pyridazine}

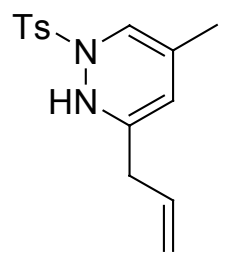

According to the general procedure outlined above using 1-(2-methylene-cyclopropyl)-but-3-en1-one-tosyl-hydrazone $(0.0165 \mathrm{~g}, 0.0570 \mathrm{mmol})$, TMEDA $(8.5 \mu \mathrm{L}, 0.0567 \mathrm{mmol})$ and $\mathrm{MgCl}_{2}$ $(0.5 \mathrm{mg}, 10 \mathrm{~mol} \%)$ in DME $(4.5 \mathrm{~mL})$ at $120{ }^{\circ} \mathrm{C}$ for 4 hours, the title compound could be obtained after flash chromatography on silica using 7:3 dichloromethane:hexanes $\left(\mathrm{R}_{f}=0.14\right)$ in $87 \%$ combined yield (0.0136 $\mathrm{g}$ of dienamine) and with a selectivity of $>20: 1$ over the azadiene product. Colorless oil. IR (neat, $\left.\mathrm{cm}^{-1}\right) 3604,2922,1698,1521,1497,1160 ; \delta_{\mathrm{H}}(400 \mathrm{MHz}$, $\left.\mathrm{CD}_{3} \mathrm{CN}\right) 8.47(\mathrm{~s}, 1 \mathrm{H}), 7.64(\mathrm{~d}, 2 \mathrm{H}, J=8.22 \mathrm{~Hz}), 7.41(\mathrm{~d}, 2 \mathrm{H}, J=8.41 \mathrm{~Hz}), 5.82(\mathrm{~m}, 1 \mathrm{H}), 5.80(\mathrm{~s}$, $1 \mathrm{H}), 5.59(\mathrm{~s}, 1 \mathrm{H}), 5.01(\mathrm{~m}, 1 \mathrm{H}), 4,98(\mathrm{~m}, 1 \mathrm{H}), 3.08(\mathrm{~d}, 2 \mathrm{H}, J=6.65 \mathrm{~Hz}), 2.46(\mathrm{~s}, 3 \mathrm{H}), 1.85(\mathrm{~s}$, 
$3 \mathrm{H}) ; \delta_{\mathrm{C}}\left(100 \mathrm{MHz}, \mathrm{CD}_{3} \mathrm{CN}\right) 160.0,146.7,136.7,136.3,131.3,129.5,119.1,118.1,116.8,108.0$, 30.7, 22.0, 12.3; $\mathrm{C}_{15} \mathrm{H}_{18} \mathrm{~N}_{2} \mathrm{O}_{2} \mathrm{~S}\left(\mathrm{MH}^{+}\right)$(ESI) Requires: 291.1161 Found: 291.1158.

Additionally, the reaction could be carried out under higher concentration $(0.028 \mathrm{M})$ using 1-(2methylene-cyclopropyl)-but-3-en-1-one-tosyl-hydrazone (0.0460 g, $0.159 \mathrm{mmol})$, TMEDA (24.0 $\mu \mathrm{L}, 0.160 \mathrm{mmol})$ and $\mathrm{MgCl}_{2}(1.5 \mathrm{mg}, 10 \mathrm{~mol} \%)$ in DME $(5.7 \mathrm{~mL})$ at $120{ }^{\circ} \mathrm{C}$ for 5 hours. The title compound could be obtained after flash chromatography on silica using 7:3 dichloromethane:hexanes $\left(\mathrm{R}_{f}=0.14\right)$ in $84 \%$ combined yield $(0.0369 \mathrm{~g}$ of dienamine $)$ and with a selectivity of $>20: 1$ over the azadiene product.

\section{3-[3-(tert-Butyl-dimethyl-silanyloxy)-propyl]-5-methyl-1- (toluene-4-sulfonyl)-1,2-dihydro-pyridazine}

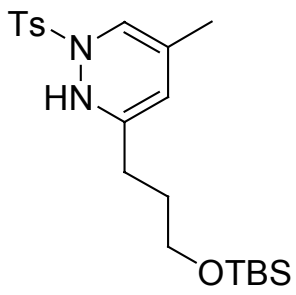

According to the general procedure outlined above using 4-(tert-butyl-dimethyl-silanyloxy)-1-(2methylene-cyclopropyl)-butan-1-one-tosyl-hydrazone $(0.0200 \mathrm{~g}, 0.0473 \mathrm{mmol})$, TMEDA (7.0 $\mu \mathrm{L}, 0.0467 \mathrm{mmol})$ and $\mathrm{MgCl}_{2}(0.5 \mathrm{mg}, 10 \mathrm{~mol} \%)$ in DME $(3.8 \mathrm{~mL})$ at $120{ }^{\circ} \mathrm{C}$ for 4 hours, the title compound could be obtained after flash chromatography on silica using 3:7 hexanes:dichloromethane $\left(\mathrm{R}_{f}=0.17\right)$ in $81 \%$ combined yield $(0.0154 \mathrm{~g}$ of dienamine $)$ and with a selectivity of $>20: 1$ over the azadiene product. Colorless oil. IR (neat, $\mathrm{cm}^{-1}$ ) 3741, 3645, 2921, $2853,1701,1650,1534,1164,1093 ; \delta_{\mathrm{H}}\left(400 \mathrm{MHz}, \mathrm{CDCl}_{3}\right) 8.42(\mathrm{~s}, 1 \mathrm{H}), 7.56(\mathrm{~d}, 2 \mathrm{H}, J=8.2$ $\mathrm{Hz}), 7.27(\mathrm{~d}, 2 \mathrm{H}, J=8.4 \mathrm{~Hz}), 6.25(\mathrm{~s}, 1 \mathrm{H}), 5.60(\mathrm{~s}, 1 \mathrm{H}), 3.45(\mathrm{t}, 2 \mathrm{H}, J=5.86 \mathrm{~Hz}), 2.44(\mathrm{~s}, 3 \mathrm{H})$, $2.08(\mathrm{t}, 2 \mathrm{H}, J=6.89 \mathrm{~Hz}), 1.97(\mathrm{~s}, 3 \mathrm{H}), 1.26(\mathrm{~m}, 2 \mathrm{H}), 0.91(\mathrm{~s}, 9 \mathrm{H}), 0.11(\mathrm{~s}, 6 \mathrm{H}) ; \delta_{\mathrm{C}}(100 \mathrm{MHz}$, $\left.\mathrm{CDCl}_{3}\right) 149.8,139.8,134.9,133.5,123.3,112.0,110.0,97.2,67.2,38.0,31.2,26.9,25.2,23.8$, 17.2, $0.00 ; \mathrm{C}_{21} \mathrm{H}_{35} \mathrm{~N}_{2} \mathrm{O}_{3} \mathrm{SSi}\left(\mathrm{MH}^{+}\right)$(ESI) Requires: 423.2132 Found: 423.2138 .

\section{3-[1,3]Dithian-2-yl-5-methyl-1-(toluene-4-sulfonyl)-1,2-dihydro-pyridazine}

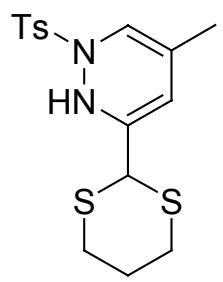

According to the general procedure outlined above using [1,3]-dithian-2-yl-(2-methylenecyclopropyl)-methanone-tosyl-hydrazone $(0.0148 \mathrm{~g}, 0.0402 \mathrm{mmol})$, TMEDA $(6.0 \mu \mathrm{L}, 0.0400$ $\mathrm{mmol})$ and $\mathrm{MgCl}_{2}(0.4 \mathrm{mg}, 10 \mathrm{~mol} \%)$ in $\operatorname{DME}(3.2 \mathrm{~mL})$ at $120{ }^{\circ} \mathrm{C}$ for 4 hours, the title compound could be obtained after flash chromatography on silica using 1:1 dichloromethane:hexanes followed by 1:9 diethyl ether:toluene $\left(\mathrm{R}_{f}=0.24\right)$ in $68 \%$ combined yield ( $0.0084 \mathrm{~g}$ of dienamine) and with a selectivity of 5:1 over the azadiene product. 
For the major dienamine product: colorless oil. IR (neat, $\mathrm{cm}^{-1}, \mathrm{~cm}^{-1}$ ) $3741,3611,3229,2922$, $2860,1698,1361,1337,1164,1089 ; \delta_{\mathrm{H}}\left(400 \mathrm{MHz}, \mathrm{CD}_{3} \mathrm{CN}\right) 8.56(\mathrm{~s}, 1 \mathrm{H}), 7.66(\mathrm{~d}, 2 \mathrm{H}, J=8.22$ $\mathrm{Hz}), 7.42$ (d, $2 \mathrm{H}, J=8.22 \mathrm{~Hz}), 5.95(\mathrm{~d}, 1 \mathrm{H}, J=4.11 \mathrm{~Hz}), 4.89(\mathrm{~s}, 1 \mathrm{H}), 2.88(\mathrm{td}, 2 \mathrm{H}, J=12.13$, $2.15 \mathrm{~Hz}), 2.76(\mathrm{dt}, 2 \mathrm{H}, J=14.48,3.72 \mathrm{~Hz}), 2.45(\mathrm{~s}, 3 \mathrm{H}), 2.04(\mathrm{~m}, 2 \mathrm{H}), 1.88(\mathrm{~s}, 3 \mathrm{H}), 1.70(\mathrm{~m}$, $3 \mathrm{H}) ; \delta_{\mathrm{C}}\left(100 \mathrm{MHz}, \mathrm{CD}_{3} \mathrm{CN}\right) 147.0,135.9,132.3,131.5,129.5,120.7,119.2,109.9,41.4,32.9$, 26.4, 22.1, 12.2; $\mathrm{C}_{16} \mathrm{H}_{21} \mathrm{~N}_{2} \mathrm{O}_{2} \mathrm{~S}_{3}\left(\mathrm{MH}^{+}\right)$(ESI) Requires: 369.0759 Found: 369.0763.

For the minor azadiene product: colorless oil, $\left(\mathrm{R}_{f}=0.3\right.$ in 1:9 diethyl ether:toluene). IR (neat, $\left.\mathrm{cm}^{-1}\right) 3604,2922,2853,1693,1647,1354,1167,1086,902 ; \delta_{\mathrm{H}}\left(400 \mathrm{MHz}, \mathrm{CD}_{3} \mathrm{CN} / \mathrm{CDCl}_{3}\right) 7.77$ $(\mathrm{d}, 2 \mathrm{H}, J=8.36 \mathrm{~Hz}), 7.42(\mathrm{~d}, 2 \mathrm{H}, J=8.06 \mathrm{~Hz}), 5.84(\mathrm{~m}, 1 \mathrm{H}), 4.76(\mathrm{~s}, 1 \mathrm{H}), 3,75(\mathrm{~s}, 1 \mathrm{H}), 2.89(\mathrm{~m}$, $4 \mathrm{H}), 2.43(\mathrm{~s}, 3 \mathrm{H}), 2.06(\mathrm{~m}, 2 \mathrm{H}), 1.86(\mathrm{~s}, 3 \mathrm{H}), 1.80(\mathrm{~m}, 3 \mathrm{H}) ; \delta_{\mathrm{C}}\left(100 \mathrm{MHz}, \mathrm{CD}_{3} \mathrm{CN}\right)$ 153.1, 146.4, 144.4, 133.3, 131.0, 130.1, 114.7, 49.7, 47.0, 30.5, 26.5, 22.0, 21.3; $\mathrm{C}_{16} \mathrm{H}_{21} \mathrm{~N}_{2} \mathrm{O}_{2} \mathrm{~S}_{3}\left(\mathrm{MH}^{+}\right)(\mathrm{ESI})$ Requires: 369.0759 Found: 369.0762 .

\section{5-Methyl-3-phenyl-1-(toluene-4-sulfonyl)-1,2-dihydro-pyridazine}

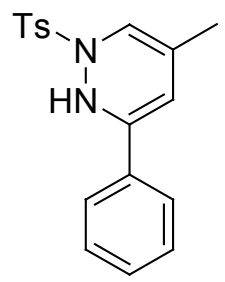

According to the general procedure outlined above using (2-methylene-cyclopropyl)-phenylmethanone-tosyl-hydrazone $(0.0150 \mathrm{~g}, 0.0460 \mathrm{mmol})$, TMEDA $(7.4 \mu \mathrm{L}, 0.0494 \mathrm{mmol})$ and $\mathrm{MgCl}_{2}(0.4 \mathrm{mg}, 10 \mathrm{~mol} \%)$ in DME $(4.6 \mathrm{~mL})$ at $120^{\circ} \mathrm{C}$ for 4 hours, the title compound could be obtained after flash chromatography on silica using 1:1 dichloromethane:hexanes in 93\% combined yield ( $0.0117 \mathrm{~g}$ of dienamine) and with a selectivity of 5:1 over the azadiene product.

For the major dienamine product: colorless oil, $\left(\mathrm{R}_{f}=0.11\right)$. IR (neat, $\left.\mathrm{cm}^{-1}\right) 3249,2921,2860$, 1340,$1164 ; \delta_{\mathrm{H}}\left(400 \mathrm{MHz}, \mathrm{CD}_{3} \mathrm{CN}\right) 8.64(\mathrm{~s}, 1 \mathrm{H}), 7.38(\mathrm{~d}, 2 \mathrm{H}, J=8.20 \mathrm{~Hz}), 7.18-7.30(\mathrm{~m}, 5 \mathrm{H})$, $7.16(\mathrm{~d}, 2 \mathrm{H}, J=8.20 \mathrm{~Hz}), 6.19(\mathrm{~s}, 1 \mathrm{H}), 6.00(\mathrm{~d}, 1 \mathrm{H}, J=1.95 \mathrm{~Hz}), 2.37(\mathrm{~s}, 3 \mathrm{H}), 1.96(\mathrm{~s}, 3 \mathrm{H}) ; \delta_{\mathrm{C}}$ $\left(100 \mathrm{MHz}, \mathrm{CDCl}_{3}\right)$ 144.9, 133.8, 133.1, 131.1, 129.6, 128.4, 128.1, 127.8, 126.7, 121.6, 118.2, 110.0, 21.8, 12.2; $\mathrm{C}_{18} \mathrm{H}_{19} \mathrm{~N}_{2} \mathrm{O}_{2} \mathrm{~S}\left(\mathrm{MH}^{+}\right)$(ESI) Requires: 327.1161 Found: 327.1168.

For the minor azadiene product: colorless oil, $\left(\mathrm{R}_{f}=0.20\right)$. IR (neat, $\left.\mathrm{cm}^{-1}\right) 3611,2915,2853$, $1698,1514,1446,1337,1157,1086 ; \delta_{\mathrm{H}}\left(400 \mathrm{MHz}, \mathrm{CD}_{3} \mathrm{CN}\right) 7.83(\mathrm{~d}, 2 \mathrm{H}, J=8.20 \mathrm{~Hz}), 7.70(\mathrm{~m}$, $2 \mathrm{H}), 7.43(\mathrm{~m}, 5 \mathrm{H}), 6.36(\mathrm{~s}, 1 \mathrm{H}), 3.81(\mathrm{~s}, 2 \mathrm{H}), 2.41(\mathrm{~s}, 3 \mathrm{H}), 1.97(\mathrm{~s}, 3 \mathrm{H}) ; \delta_{\mathrm{C}}\left(100 \mathrm{MHz}, \mathrm{CD}_{3} \mathrm{CN}\right)$ 151.0, 145.2, 143.0, 135.2, 132.4, 130.1, 130.0, 129.0, 128.9, 126.0, 113.3, 45.8, 20.9, 20.5; $\mathrm{C}_{18} \mathrm{H}_{19} \mathrm{~N}_{2} \mathrm{O}_{2} \mathrm{~S}\left(\mathrm{MH}^{+}\right)$(ESI) Requires: 327.1161 Found: 327.1173 .

Additionally, the reaction could be carried out under higher concentration $(0.028 \mathrm{M})$ using (2methylene-cyclopropyl)-phenyl-methanone-tosyl-hydrazone (0.0496 g, $0.152 \mathrm{mmol})$, TMEDA $(22.8 \mu \mathrm{L}, 0.152 \mathrm{mmol})$ and $\mathrm{MgCl}_{2}(1.5 \mathrm{mg}, 10 \mathrm{~mol} \%)$ in DME $(5.4 \mathrm{~mL})$ at $120{ }^{\circ} \mathrm{C}$ for 5 hours, the title compound could be obtained after flash chromatography on silica using 1:1 dichloromethane:hexanes in $91 \%$ combined yield ( $0.0378 \mathrm{~g}$ of dienamine) and with a selectivity of 5:1 over the azadiene product. 


\section{3-Furan-2-yl-5-methyl-1-(toluene-4-sulfonyl)-1,2-dihydro-pyridazine}

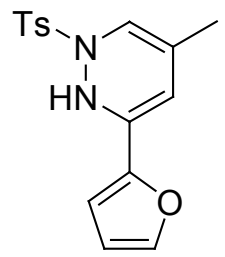

According to the general procedure outlined above using furan-2-yl-(2-methylene-cyclopropyl)methanone-tosyl-hydrazone $(0.0160 \mathrm{~g}, 0.0507 \mathrm{mmol})$, TMEDA $(7.6 \mu \mathrm{L}, 0.0507 \mathrm{mmol})$ and $\mathrm{MgCl}_{2}(0.5 \mathrm{mg}, 10 \mathrm{~mol} \%)$ in DME $(4.1 \mathrm{~mL})$ at $120^{\circ} \mathrm{C}$ for 4 hours, the title compound could be obtained after flash chromatography on silica using 1:3 ethyl acetate:hexanes in $81 \%$ combined yield ( $0.0122 \mathrm{~g}$ of dienamine) and with a selectivity of $15: 1$ over the azadiene product. Colorless oil. $\left(\mathrm{R}_{f}=0.3\right)$. IR (neat, $\left.\mathrm{cm}^{-1}\right) 3611,2922,2846,1715,1354,1164,1086,998 ; \delta_{\mathrm{H}}(400 \mathrm{MHz}$, $\left.\mathrm{CDCl}_{3}\right) 7.81(\mathrm{~s}, 1 \mathrm{H}), 7.36(\mathrm{~d}, 2 \mathrm{H}, J=8.36 \mathrm{~Hz}), 7.19(\mathrm{~m}, 1 \mathrm{H}), 7.08(\mathrm{~d}, 2 \mathrm{H}, J=8.06 \mathrm{~Hz}), 6.58(\mathrm{~m}$, $1 \mathrm{H}), 6.18(\mathrm{dd}, 1 \mathrm{H}, J=3.37,1.91 \mathrm{~Hz}), 6.02(\mathrm{~d}, 1 \mathrm{H}, J=1.90 \mathrm{~Hz}), 5.90(\mathrm{~d}, 1 \mathrm{H}, J=2.93 \mathrm{~Hz}), 2.35$ $(\mathrm{s}, 3 \mathrm{H}), 2.04(\mathrm{~s}, 3 \mathrm{H}) ; \delta_{\mathrm{C}}\left(100 \mathrm{MHz}, \mathrm{CDCl}_{3}\right)$ 146.1, 144.9, 140.8, 133.0, 129.7, 128.2, 123.4, 121.4, 118.1, 111.3, 109.1, 105.4, 21.8, 12.1; $\mathrm{C}_{16} \mathrm{H}_{16} \mathrm{~N}_{2} \mathrm{O}_{3} \mathrm{~S}\left(\mathrm{MH}^{+}\right)$(ESI) Requires: 317.0954 Found: 317.0951.

\section{3,5-Dimethyl-2H-[1,2]oxazine \\ and}

3,5-Dimethyl-6H-[1,2]oxazine
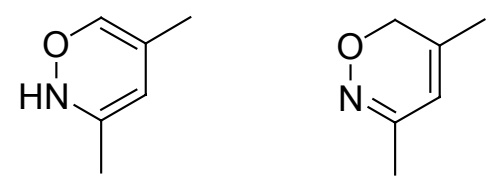

According to the general procedure outlined above using 1-(2-methylene-cyclopropyl)-ethanone oxime (0.0329 g, $0.296 \mathrm{mmol})$, TMEDA (45.0 $\mu \mathrm{L}, 0.300 \mathrm{mmol})$ and $\mathrm{MgCl}_{2}(2.8 \mathrm{mg}, 10 \mathrm{~mol} \%)$ in DME (5.9 mL) at $120{ }^{\circ} \mathrm{C}$ for 18 hours, the title compound could be obtained after flash chromatography on silica using 1:4 diethyl ether:hexanes $\left(\mathrm{R}_{f}=0.31\right)$ in $73 \%$ combined yield $(0.0240 \mathrm{~g})$ as a inseparable 3:1 mixture of the two regioisomers (major being the $2 \mathrm{H}$-isomer) and as a clear colourless oil. IR (neat, $\mathrm{cm}^{-1}$ ) 3142, 2923, 1621, 1537, 1441, 1383, 1334, 1204, 1139,1035, 907, 784, 767, 704; $\delta_{\mathrm{H}}\left(400 \mathrm{MHz}, \mathrm{CDCl}_{3}\right) 9.20(\mathrm{bs}, 0.75 \mathrm{H}), 6.37(\mathrm{~s}, 0.75 \mathrm{H}), 5.98(\mathrm{~s}$, $0.25 \mathrm{H}), 5.49(\mathrm{~s}, 0.75 \mathrm{H}), 3.87(\mathrm{~s}, 0.50 \mathrm{H}), 2.13(\mathrm{~s}, 2.25 \mathrm{H}), 2.07(\mathrm{~s}, 0.75 \mathrm{H}), 1.99(\mathrm{~s}, 2.25 \mathrm{H}), 1.96(\mathrm{~s}$, $0.75) ; \delta_{\mathrm{C}}\left(100 \mathrm{MHz}, \mathrm{CDCl}_{3}\right) 153.6,145.4,125.4,123.4,113.9,113.1,102.8,68.8,14.5,12.2$, 11.7, 10.4; $\mathrm{C}_{6} \mathrm{H}_{10} \mathrm{NO}\left(\mathrm{MH}^{+}\right)(\mathrm{ESI})$ Requires: 112.0756 Found: 112.0749. 


\section{General Procedure for the Azadiene}

To an oven-dried flask, was added the MCP hydrazone (1 equiv), $\operatorname{MgI}_{2}$ ( 1 equiv) and $\mathrm{N}$ (benzylidene)-toluenesulfonamide (1.2 equiv). THF $(0.02 \mathrm{M})$ was then added and the solution was stirred vigorously for 25 minutes at room temperature. The resulting white suspension was then heated to reflux (oil bath temperature $=86^{\circ} \mathrm{C}$ ) until judged complete by TLC. The resulting solution was cooled and quenched by the addition of a saturated sodium sulfite solution. The reaction solution was stirred for 20 minutes, extracted with ethyl acetate $(3 \times 50 \mathrm{~mL})$, and the combined organic layers were washed with brine, dried over $\mathrm{Na}_{2} \mathrm{SO}_{4}$ and concentrated in vacuo. The crude mixture was then purified by chromatography on silica to afford the title compound.

\section{3,5-Dimethyl-1-(toluene-4-sulfonyl)-1,6-dihydro-pyridazine}

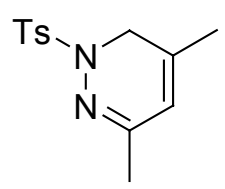

According to the general procedure outlined above using 1-(2-methylene-cyclopropyl)-ethanonetosyl-hydrazone $(0.0270 \mathrm{~g}, 0.101 \mathrm{mmol}), \mathrm{N}$-(benzylidene)-toluenesulfonamide $(0.0318 \mathrm{~g}, 0.123$ $\mathrm{mmol}), \mathrm{MgI}_{2}(0.0285 \mathrm{~g}, 0.102 \mathrm{mmol})$ in THF $(5.1 \mathrm{~mL})$ for 15 hours, the title compound could be obtained after flash chromatography on silica using 1:2 ethyl acetate:hexanes $\left(\mathrm{R}_{f}=0.4\right)$ in $86 \%$ yield $(0.0232 \mathrm{~g})$ and with a selectivity of $>20: 1$ over the dienamine product. Clear crystals, $\mathrm{mp}$ $=138-140{ }^{\circ} \mathrm{C}\left(\mathrm{CH}_{2} \mathrm{Cl}_{2}\right)$. IR (neat, $\left.\mathrm{cm}^{-1}\right) 2914,2809,1662,1595,1493,1441,1223,1121,1090$, 1033, 1010, 946, 901, 881, 814, 706, 665; $\delta_{\mathrm{H}}\left(400 \mathrm{MHz}, \mathrm{CDCl}_{3}\right) 7.80$ (d, 2H, $\left.J=8.4 \mathrm{~Hz}\right), 7.33$ $(\mathrm{d}, 2 \mathrm{H}, J=7.9 \mathrm{~Hz}), 5.59(\mathrm{~s}, 1 \mathrm{H}), 3.58(\mathrm{~s}, 2 \mathrm{H}), 2.43(\mathrm{~s}, 3 \mathrm{H}), 1.94(\mathrm{~s}, 3 \mathrm{H}), 1.85(\mathrm{~s}, 3 \mathrm{H}) ; \delta_{\mathrm{C}}(125$ $\left.\mathrm{MHz}, \mathrm{CDCl}_{3}\right) 151.8,144.2,140.8,132.4,129.6,129.0,116.6,45.5,21.8,21.7,20.8$; $\mathrm{C}_{13} \mathrm{H}_{16} \mathrm{~N}_{2} \mathrm{O}_{2} \mathrm{~S}\left(\mathrm{M}^{+}\right)$(EI) Requires: 264.0933 Found: 264.0930.

\section{5-Methyl-3-phenyl-1-(toluene-4-sulfonyl)-1,6-dihydro-pyridazine}

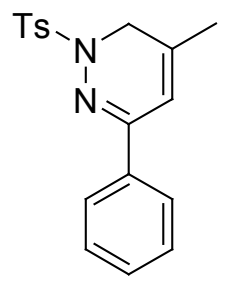

According to the general procedure outlined above using (2-methylene-cyclopropyl)-phenylmethanone-tosyl-hydrazone $(0.0417 \mathrm{~g}, 0.128 \mathrm{mmol}), \quad N$-(benzylidene)-toluenesulfonamide $(0.0390 \mathrm{~g}, 0.150 \mathrm{mmol}), \mathrm{MgI}_{2}(0.0350 \mathrm{~g}, 0.126 \mathrm{mmol})$ in THF $(6.4 \mathrm{~mL})$ for 3 hours, the title compound could be obtained after flash chromatography on silica using 1:3 THF:hexanes $\left(\mathrm{R}_{f}=\right.$ $0.31)$ in $72 \%$ yield $(0.0300 \mathrm{~g})$ and with a selectivity of $>20: 1$ over the dienamine product. 


\section{Mechanistic Studies}

\section{3,3-Dideuterio-2-methylene-cyclopropanecarboxylic acid}

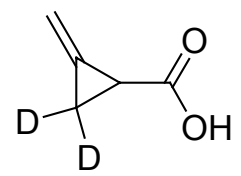

This compound was prepared using Lautens' procedure for the samarium-mediated cyclopropanation of allenic alcohols. ${ }^{5}$ To a slurry of samarium metal $(14.5 \mathrm{~g}, 96.4 \mathrm{mmol})$ in THF $(387 \mathrm{~mL})$ at room temperature, was added $\mathrm{HgCl}_{2}(2.62 \mathrm{~g}, 9.65 \mathrm{mmol})$. The reaction was stirred for 30 minutes at room temperature, after which a solution of buta-2,3-dien-1-ol (1.23 g, $17.5 \mathrm{mmol})$ in THF $(252 \mathrm{~mL})$ was added. The resulting slurry was stirred for an additional 25 minutes at room temperature before being cooled to $-78{ }^{\circ} \mathrm{C} . \mathrm{CD}_{2} \mathrm{I}_{2}(26.2 \mathrm{~g}, 97.1 \mathrm{mmol})$ was then added dropwise over the period of several hours. The resulting blue solution was allowed to warm slowly to room temperature and was stirred overnight before being quenched with a saturated aqueous $\mathrm{K}_{2} \mathrm{CO}_{3}$ solution. The slurry was then extracted with ether $(3 \times 75 \mathrm{~mL})$ and the combined organic layers were washed with brine, dried over $\mathrm{Na}_{2} \mathrm{SO}_{4}$ and concentrated in vacuo. The crude mixture was passed through a short plug of silica to remove any remaining metals using 1:1 diethyl ether:pentane to afford the 3,3-dideuterio-2-methylenecyclopropyl alcohol (approximately $0.878 \mathrm{~g}, 58 \%$ NMR yield) which was used in the next step without further purification.

To a solution of the 3,3-dideuterio-2-methylenecyclopropyl alcohol (0.878 g, $10.2 \mathrm{mmol})$ in acetone $(17.3 \mathrm{~mL})$ at $-10{ }^{\circ} \mathrm{C}$, was slowly added a solution of $\mathrm{CrO}_{3}(4.00 \mathrm{~g}, 40.0 \mathrm{mmol})$ in concentrated sulfuric acid $(4.2 \mathrm{~mL})$ and water $(12 \mathrm{~mL})$ until the reaction was judged complete. The reaction was then quenched with saturated sodium sulfite and dichloromethane $(50 \mathrm{~mL})$ and water $(30 \mathrm{~mL})$ were added. The resulting organic layer was removed, and the aqueous layer was washed with dichloromethane $(3 \times 75 \mathrm{~mL})$. The combined organic layers were washed with brine, dried over $\mathrm{Na}_{2} \mathrm{SO}_{4}$ and concentrated in vacuo. The crude mixture was then purified by flash chromatography on silica using 1:1 diethyl ether:pentane $\left(\mathrm{R}_{f}=0.48\right)$ followed by additional purification via an acid/base extraction to afford the title compound in $43 \%$ yield over two steps (74\% yield on the oxidation) $(0.758 \mathrm{~g}) . \delta_{\mathrm{H}}\left(400 \mathrm{MHz}, \mathrm{CDCl}_{3}\right) 5.57(\mathrm{~s}, 2 \mathrm{H}), 2.26(\mathrm{~s}, 1 \mathrm{H}) ; \delta_{\mathrm{D}}(46$ $\left.\mathrm{MHz}, \mathrm{CHCl}_{3}\right) 1.86$ (bs, 1D), 1.69 (bs, 1D).

\section{3,3-Dideuterio-2-methylene-cyclopropanecarboxylic acid methoxy-methyl-amide}

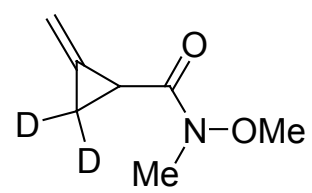

The title compound was prepared in $93 \%$ yield $(0.200 \mathrm{~g}, 1.40 \mathrm{mmol})$ according to the procedure outlined on page SI-3 using 3,3-dideuterio-2-methylene-cyclopropanecarboxylic acid (0.150 g, $1.50 \mathrm{mmol}) . \delta_{\mathrm{H}}\left(400 \mathrm{MHz}, \mathrm{CDCl}_{3}\right) 5.52(\mathrm{~d}, 1 \mathrm{H}, J=2.5 \mathrm{~Hz}), 5.46(\mathrm{~s}, 1 \mathrm{H}), 3.78(\mathrm{~s}, 3 \mathrm{H}), 3.23(\mathrm{~s}$, $3 \mathrm{H}), 2.80$ (bs, 1H); $\delta_{\mathrm{D}}\left(46 \mathrm{MHz}, \mathrm{CHCl}_{3}\right) 1.86$ (bs, 1D), 1.59 (bs, 1D). 
1-(3,3-Dideuterio-2-methylene-cyclopropyl)-ethanone-tosyl-hydrazone

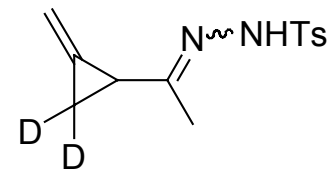

The title compound was prepared as a $2.3: 1$ mixture in $68 \%$ yield over two steps $(0.254 \mathrm{~g}, 0.954$ $\mathrm{mmol}$ ) according to the procedure outlined on page SI-4 using 3,3-dideuterio-2-methylenecyclopropanecarboxylic acid methoxy-methyl-amide $(0.200 \mathrm{~g}, 1.40 \mathrm{mmol}) . \quad \delta_{\mathrm{H}}(400 \mathrm{MHz}$, $\left.\mathrm{CDCl}_{3}\right) 7.37(\mathrm{~d}, 1.4 \mathrm{H}, J=8.2 \mathrm{~Hz}), 7.36(\mathrm{~d}, 0.7 \mathrm{H}, J=8.2 \mathrm{~Hz}), 7.57(\mathrm{bs}, 0.3 \mathrm{H}), 7.33(\mathrm{~d}, 2 \mathrm{H}, J=$ $8.0 \mathrm{~Hz}), 7.14(\mathrm{bs}, 0.7 \mathrm{H}), 5.54(\mathrm{~d}, 0.3 \mathrm{H}, J=2.3 \mathrm{~Hz}), 5.52(\mathrm{~d}, 0.7 \mathrm{H}, J=2.3 \mathrm{~Hz}), 5.49(\mathrm{~d}, 0.3 \mathrm{H}, J=$ $1.8 \mathrm{~Hz}), 5.46(\mathrm{~d}, 0.7 \mathrm{H}, J=1.6 \mathrm{~Hz}), 2.44(\mathrm{~s}, 3 \mathrm{H}), 2.39$ (bs, $0.7 \mathrm{H}), 2.25$ (bs, $0.3 \mathrm{H}), 1.71(\mathrm{~s}, 0.9 \mathrm{H})$, $1.49(\mathrm{~s}, 2.1 \mathrm{H}) ; \delta_{\mathrm{D}}\left(46 \mathrm{MHz}, \mathrm{CHCl}_{3}\right) 1.31(\mathrm{bs}, 2 \mathrm{D})$.

5-dideuteriomethyl-3-methyl-1-(toluene-4-sulfonyl)-1,2-dihydro-pyridazine

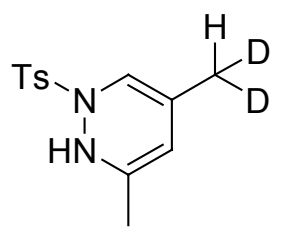

The title compound was prepared in $77 \%$ yield $(0.0154 \mathrm{~g}, 0.0578 \mathrm{mmol})$ according to the procedure outlined on page SI-9 using 1-(3,3-dideuterio-2-methylene-cyclopropyl)-ethanonetosyl-hydrazone $(0.0199 \mathrm{~g}, 0.0747 \mathrm{mmol}) . \quad \delta_{\mathrm{H}}\left(400 \mathrm{MHz}, \mathrm{CDCl}_{3}\right) 7.67(\mathrm{~d}, 2 \mathrm{H}, J=8.2 \mathrm{~Hz}), 7.31$ $(\mathrm{d}, 2 \mathrm{H}, J=8.0 \mathrm{~Hz}), 6.99(\mathrm{bs}, 1 \mathrm{H}), 5.98(\mathrm{~s}, 1 \mathrm{H}), 5.60(\mathrm{~s}, 1 \mathrm{H}), 2.46(\mathrm{~s}, 3 \mathrm{H}), 1.92(\mathrm{~s}, 1 \mathrm{H}), 1.87(\mathrm{~s}$, $3 \mathrm{H}) ; \delta_{\mathrm{D}}\left(46 \mathrm{MHz}, \mathrm{CHCl}_{3}\right) 1.91(\mathrm{bs}, 2 \mathrm{D})$. 

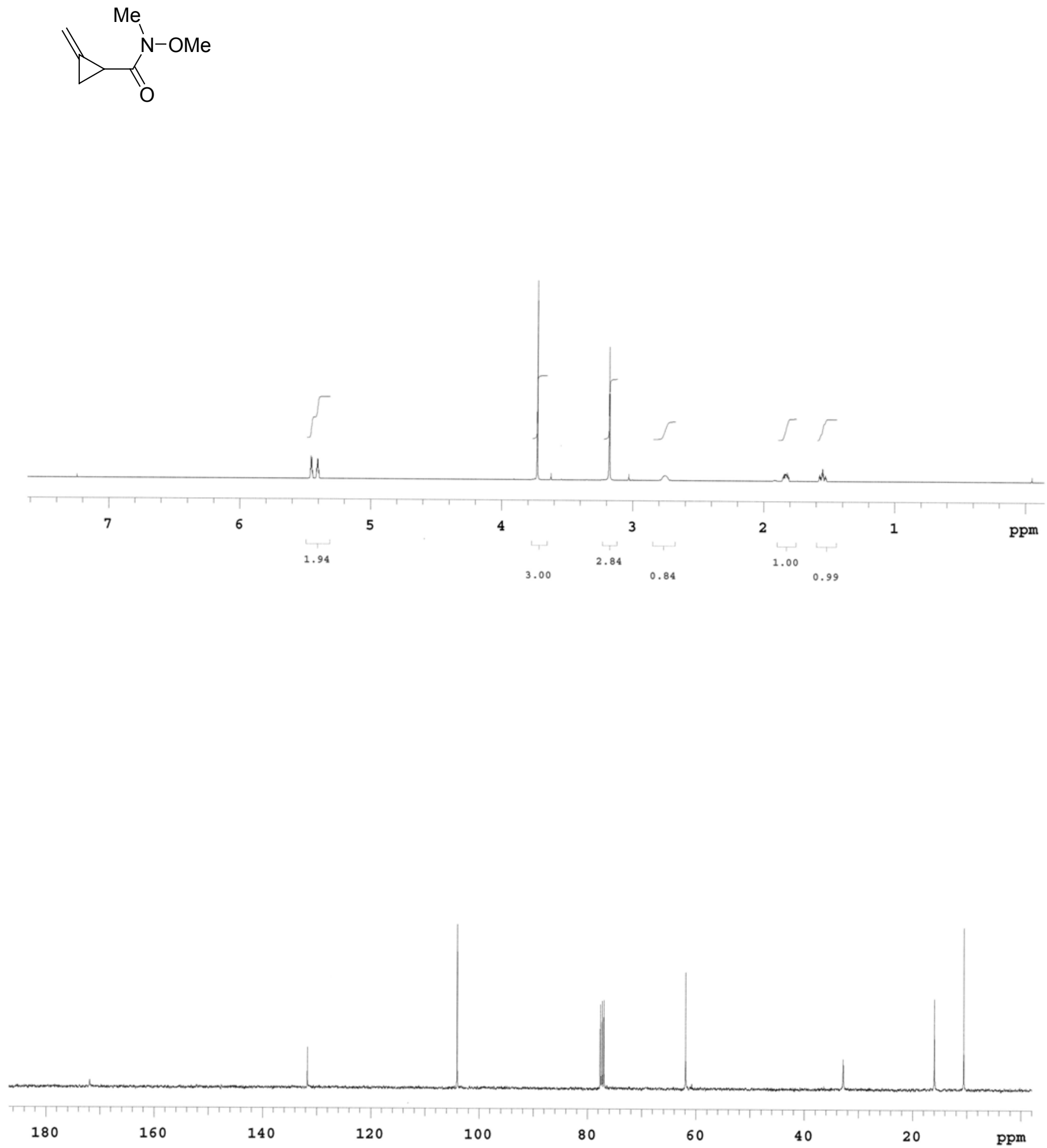


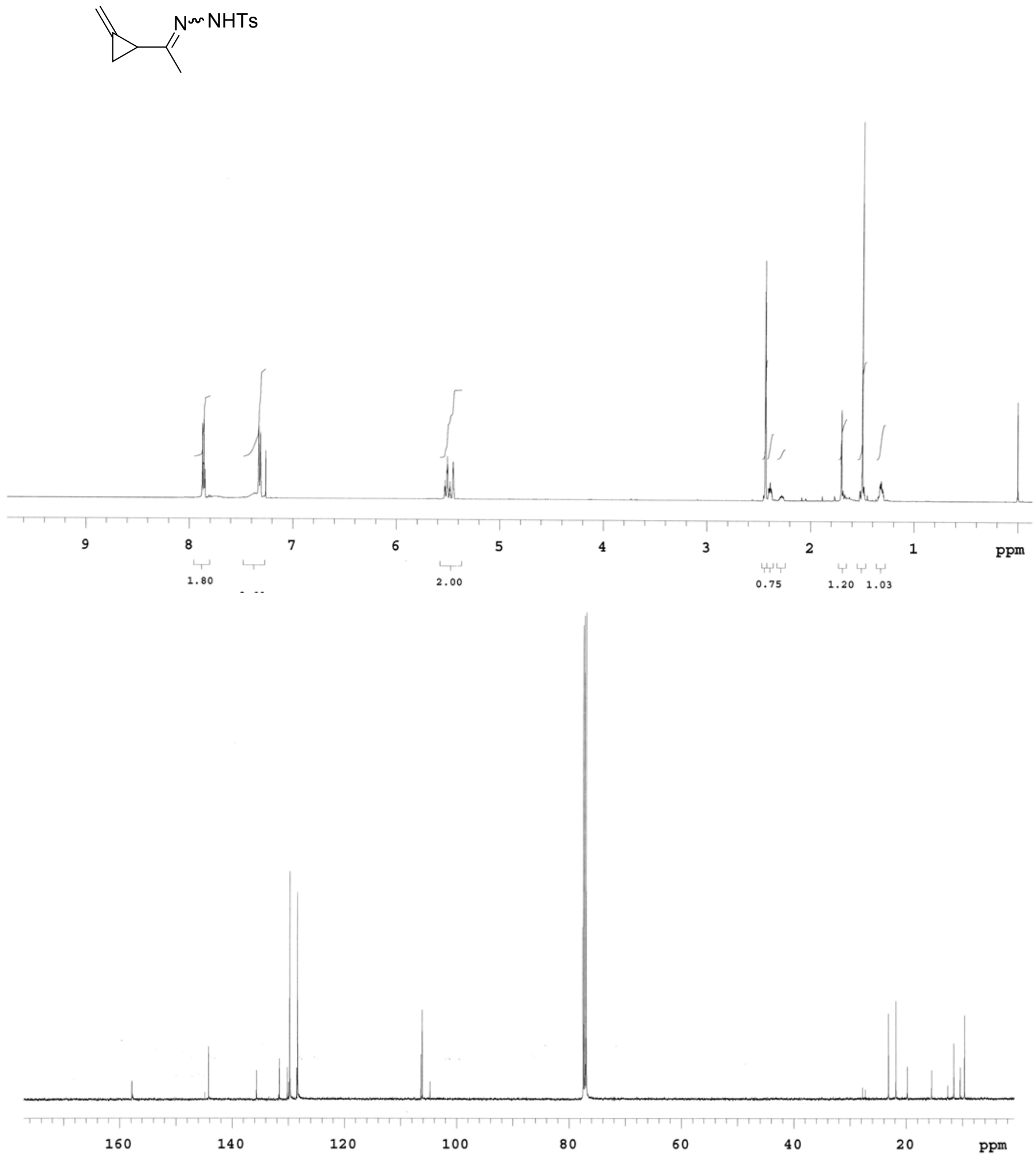



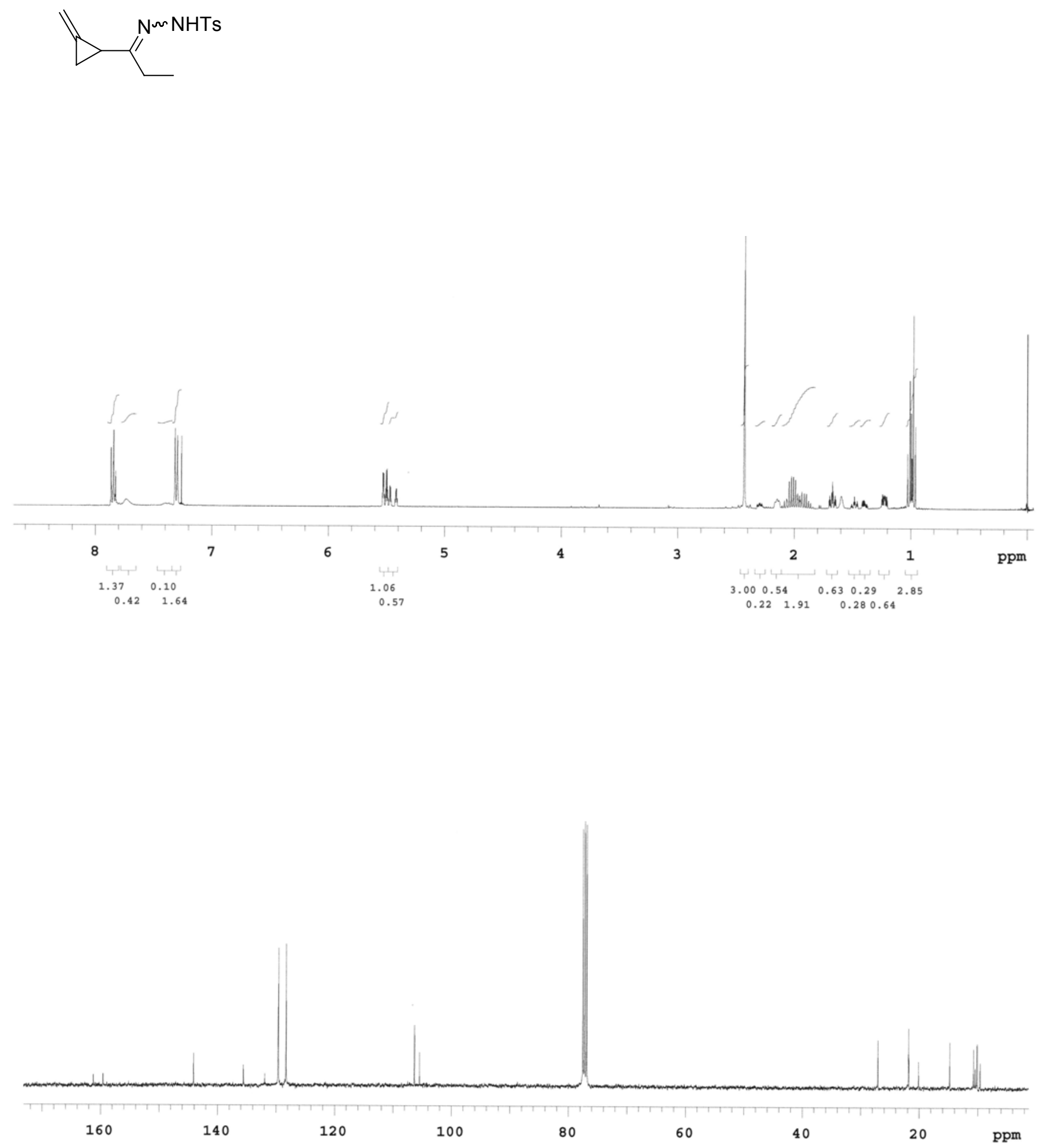

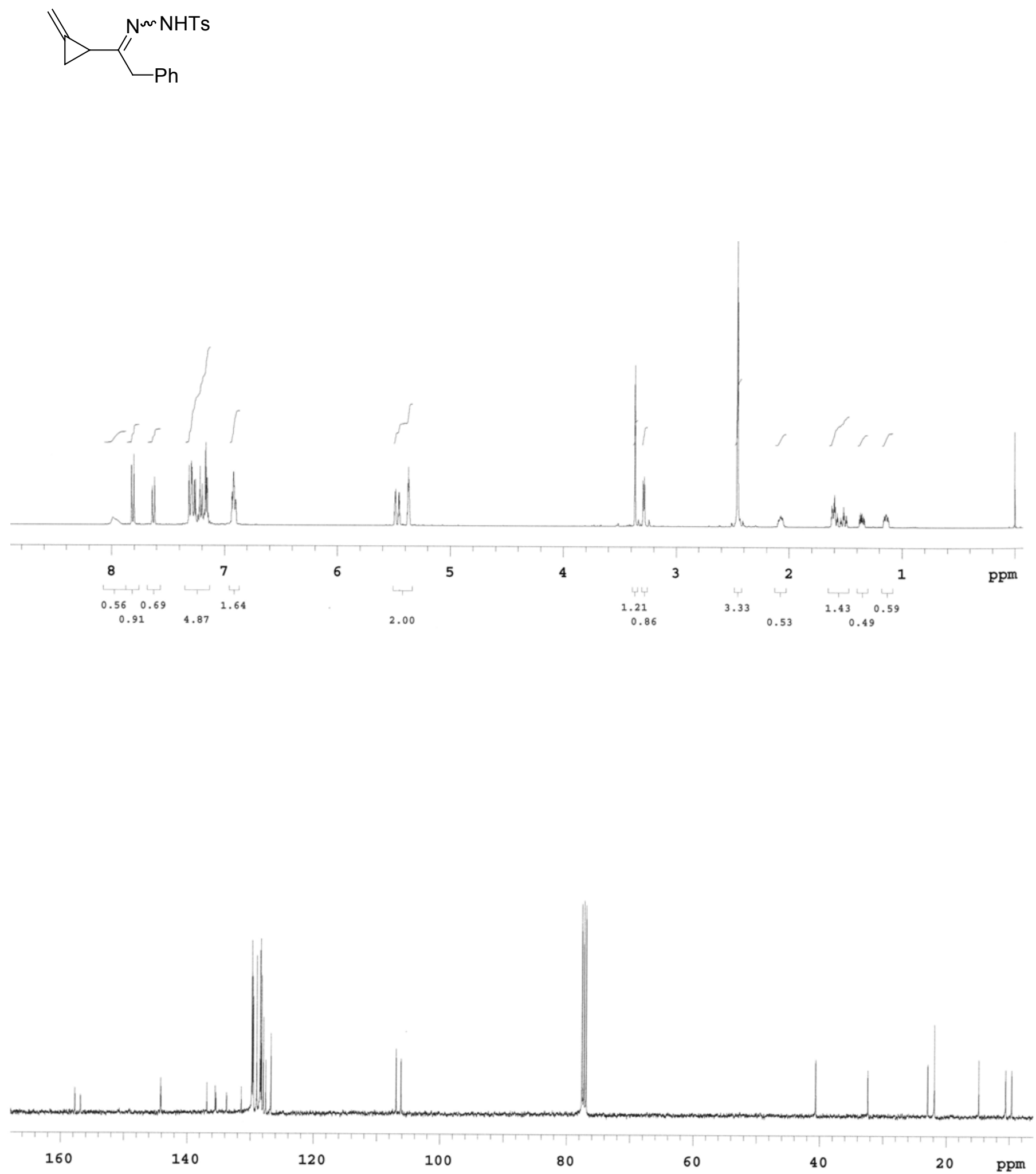

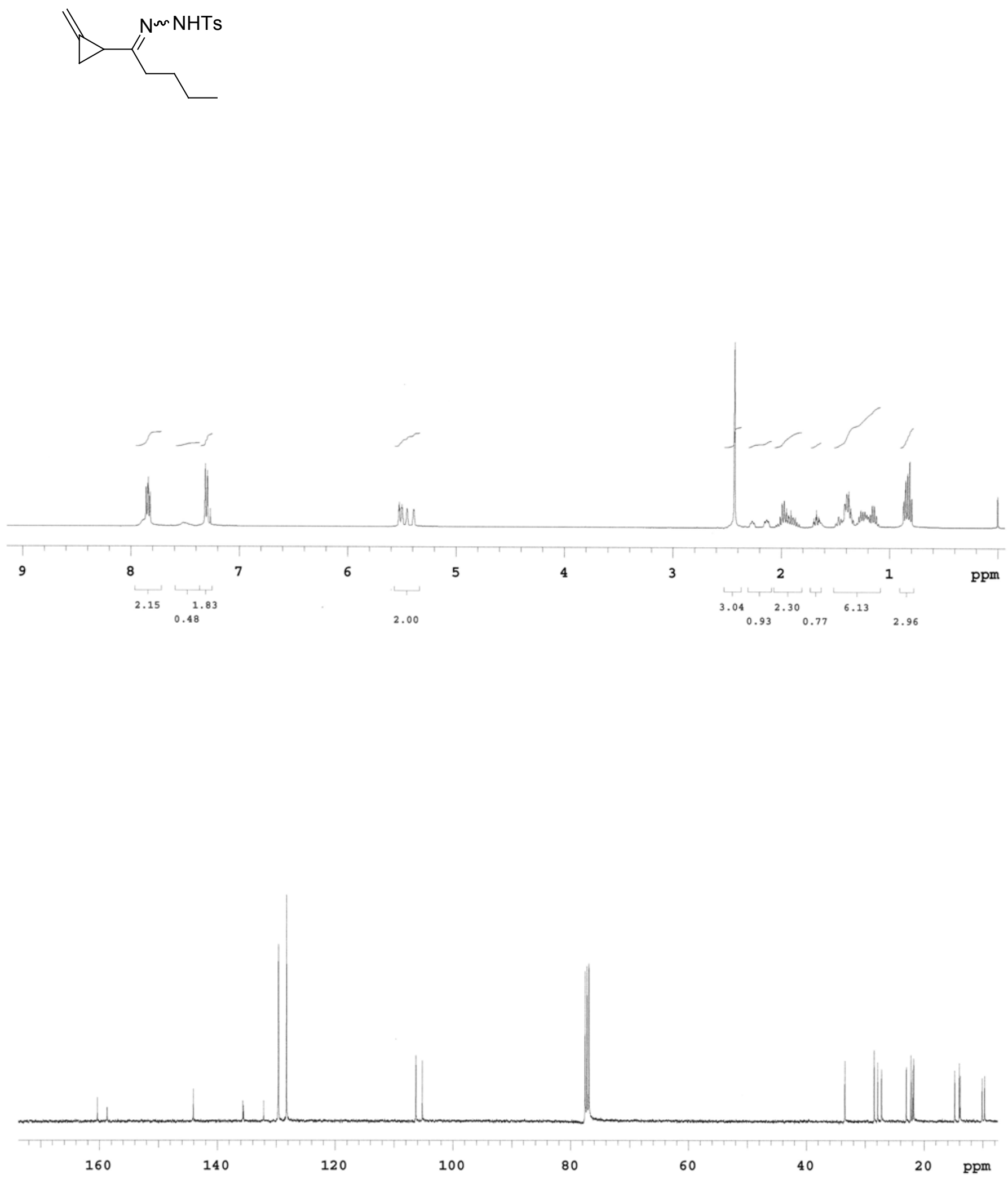

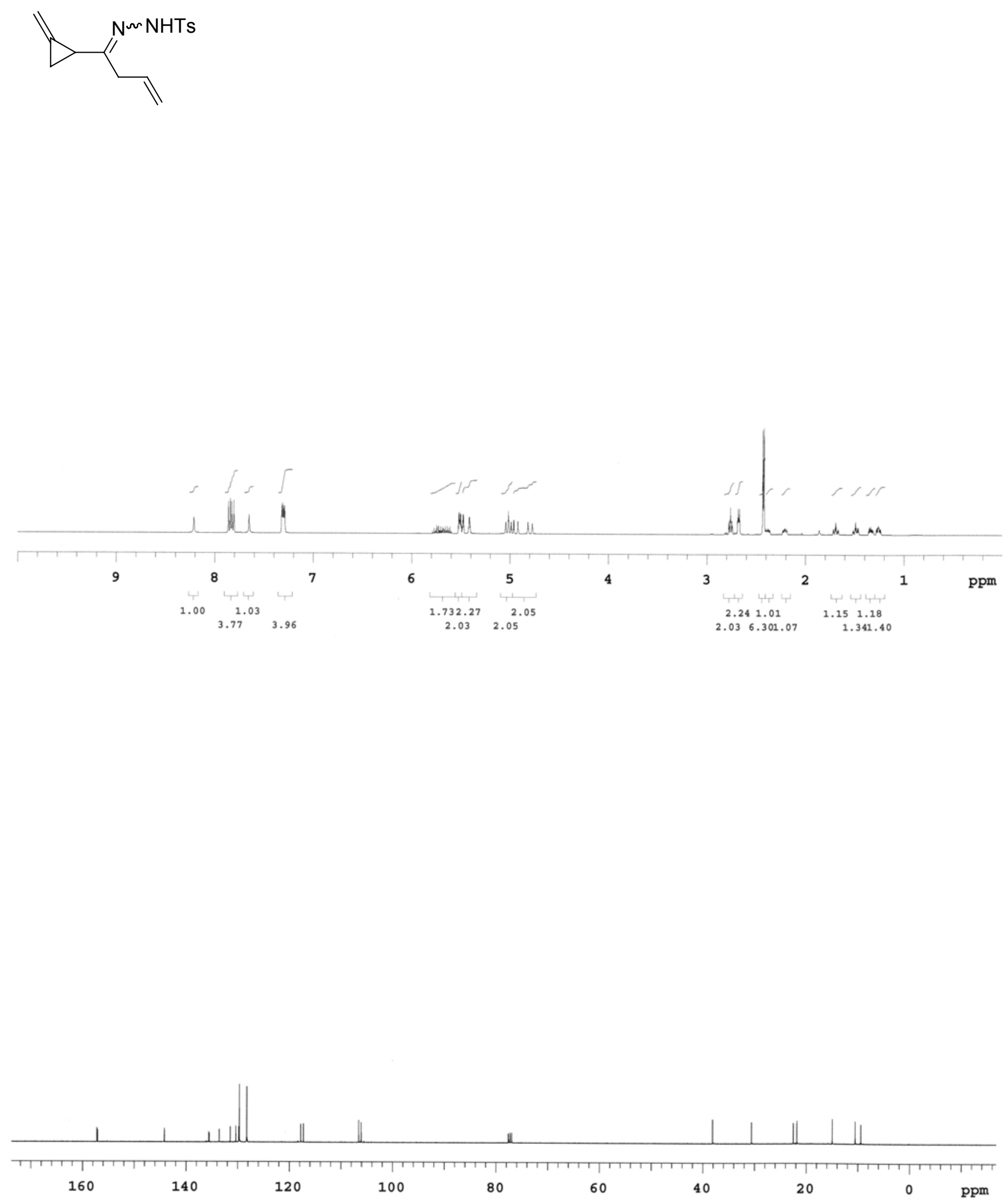

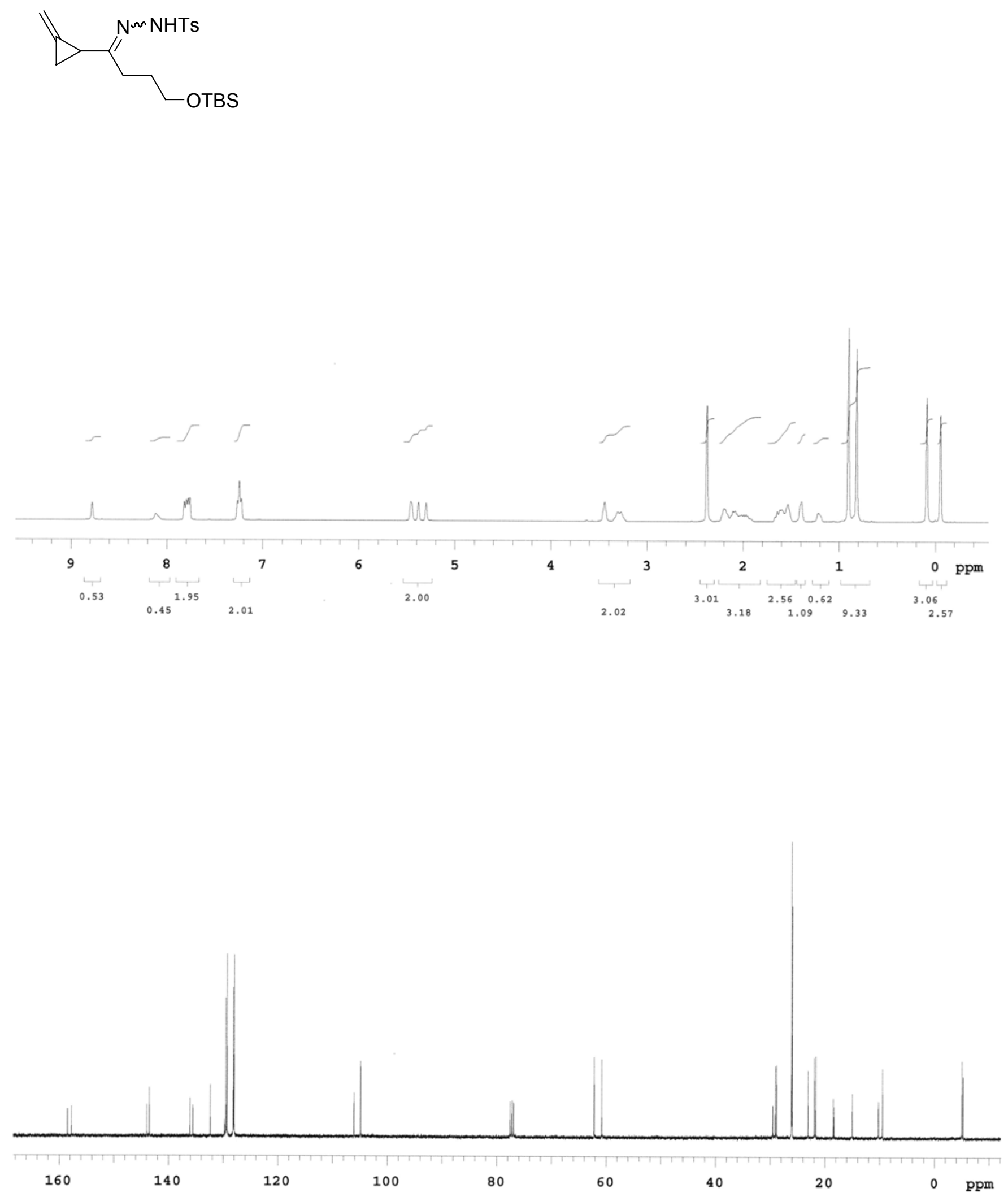

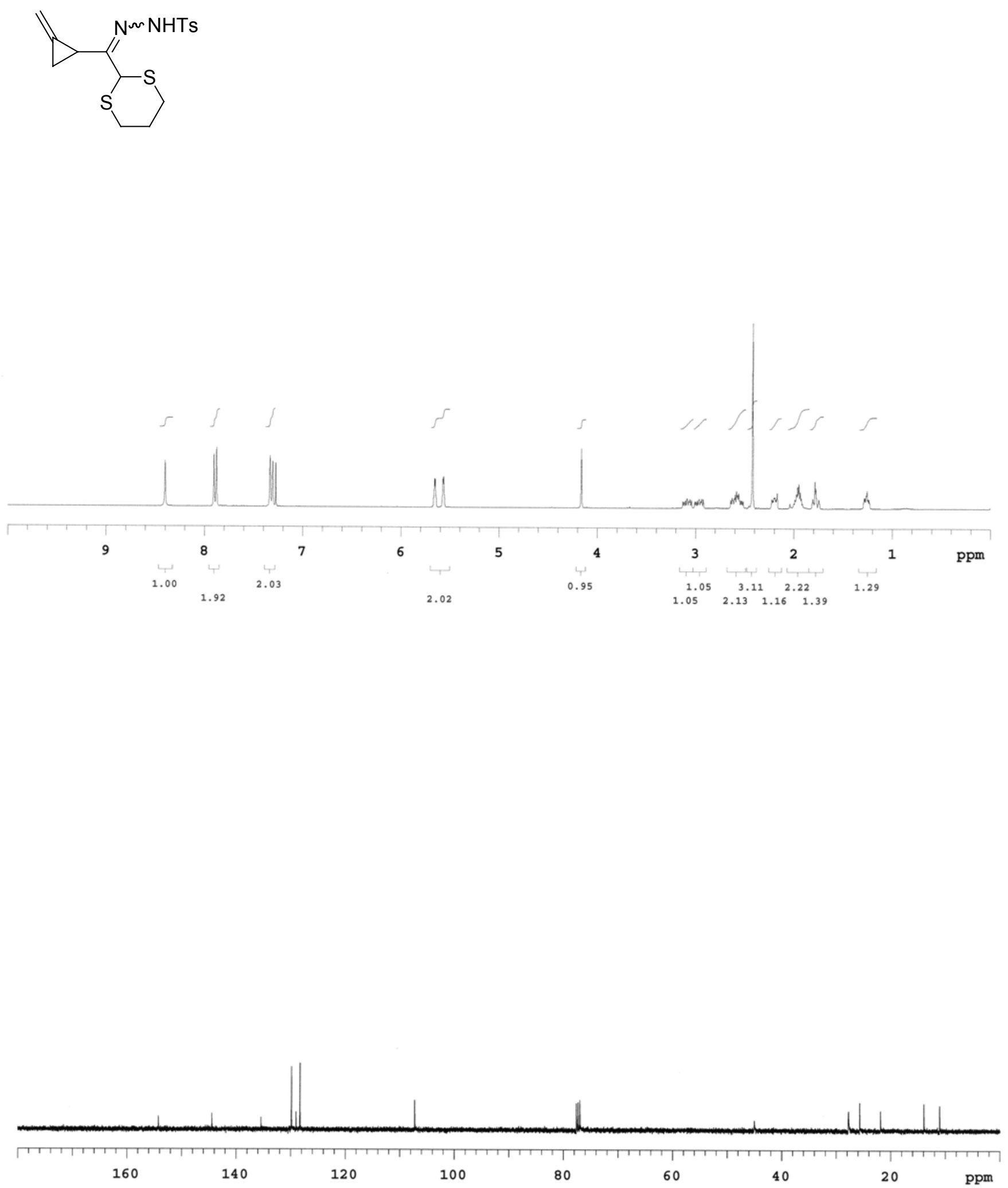


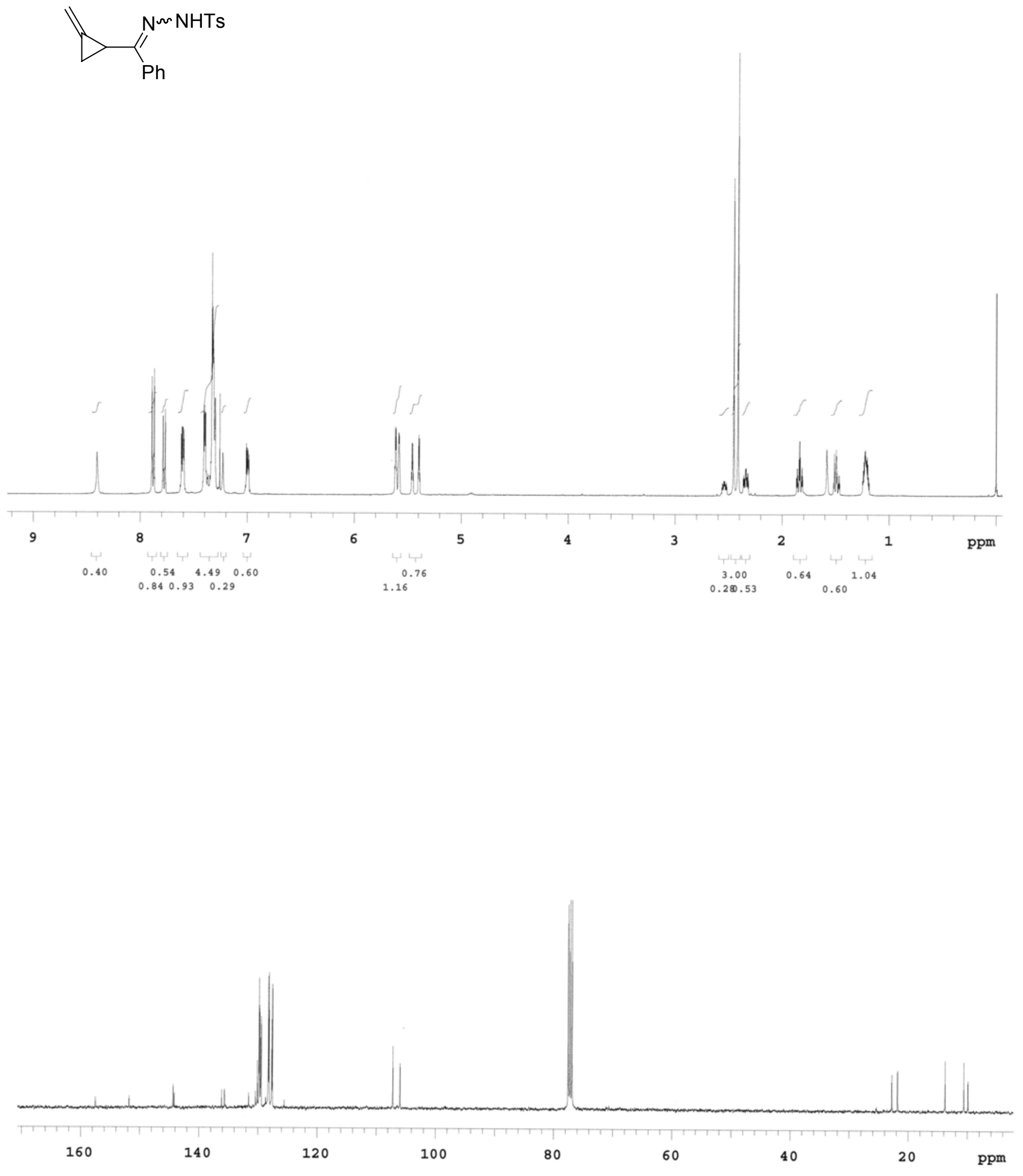



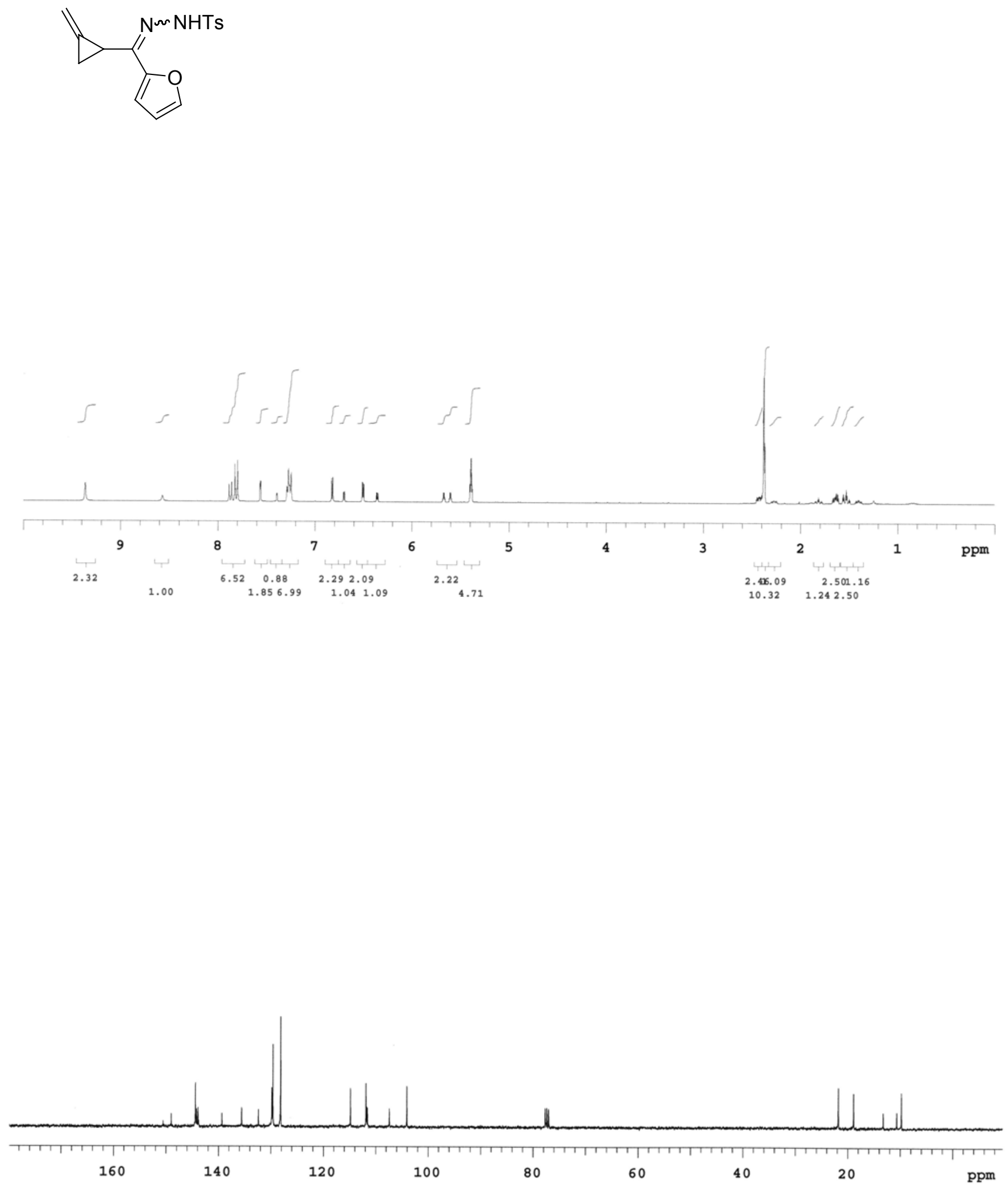

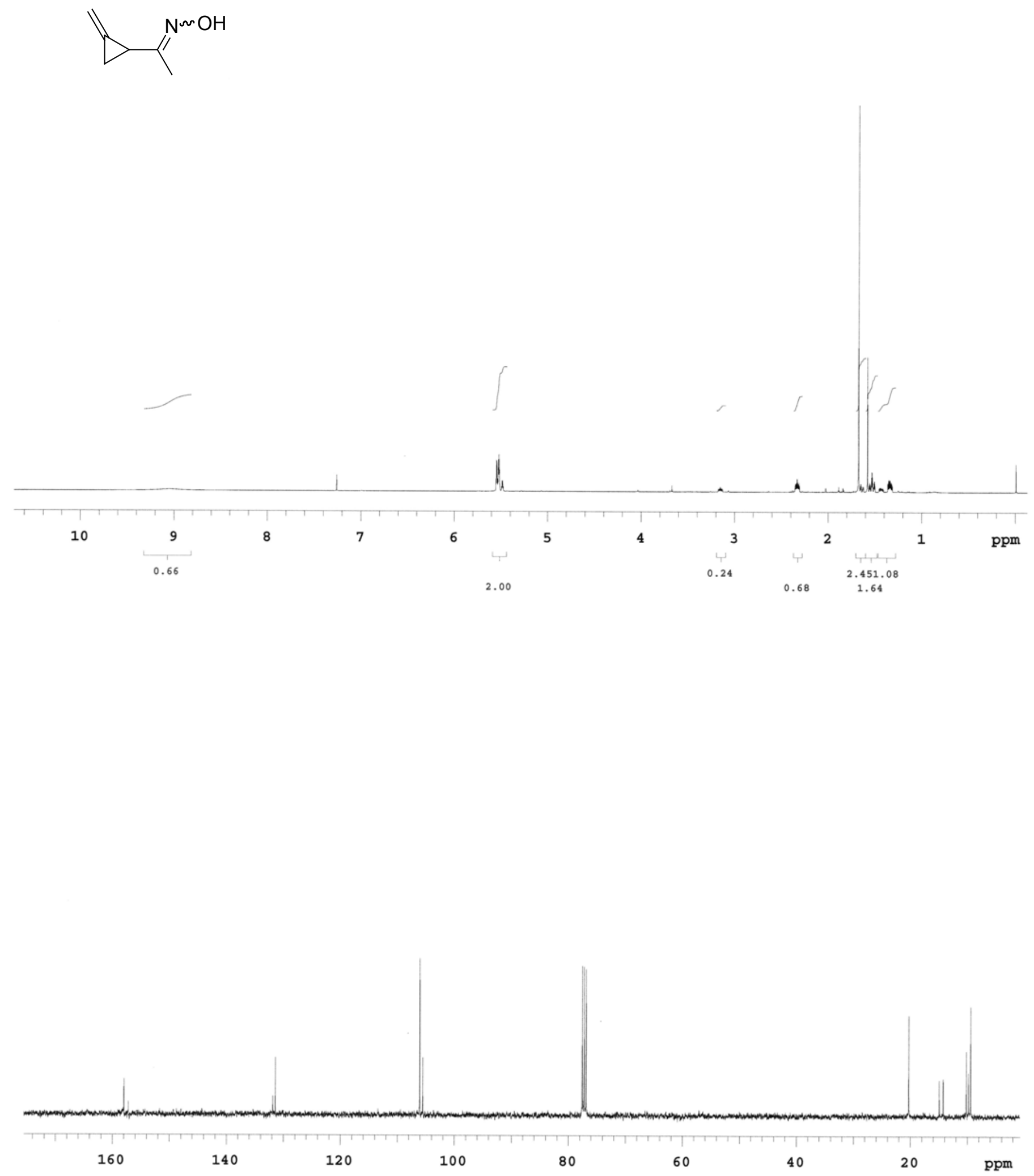

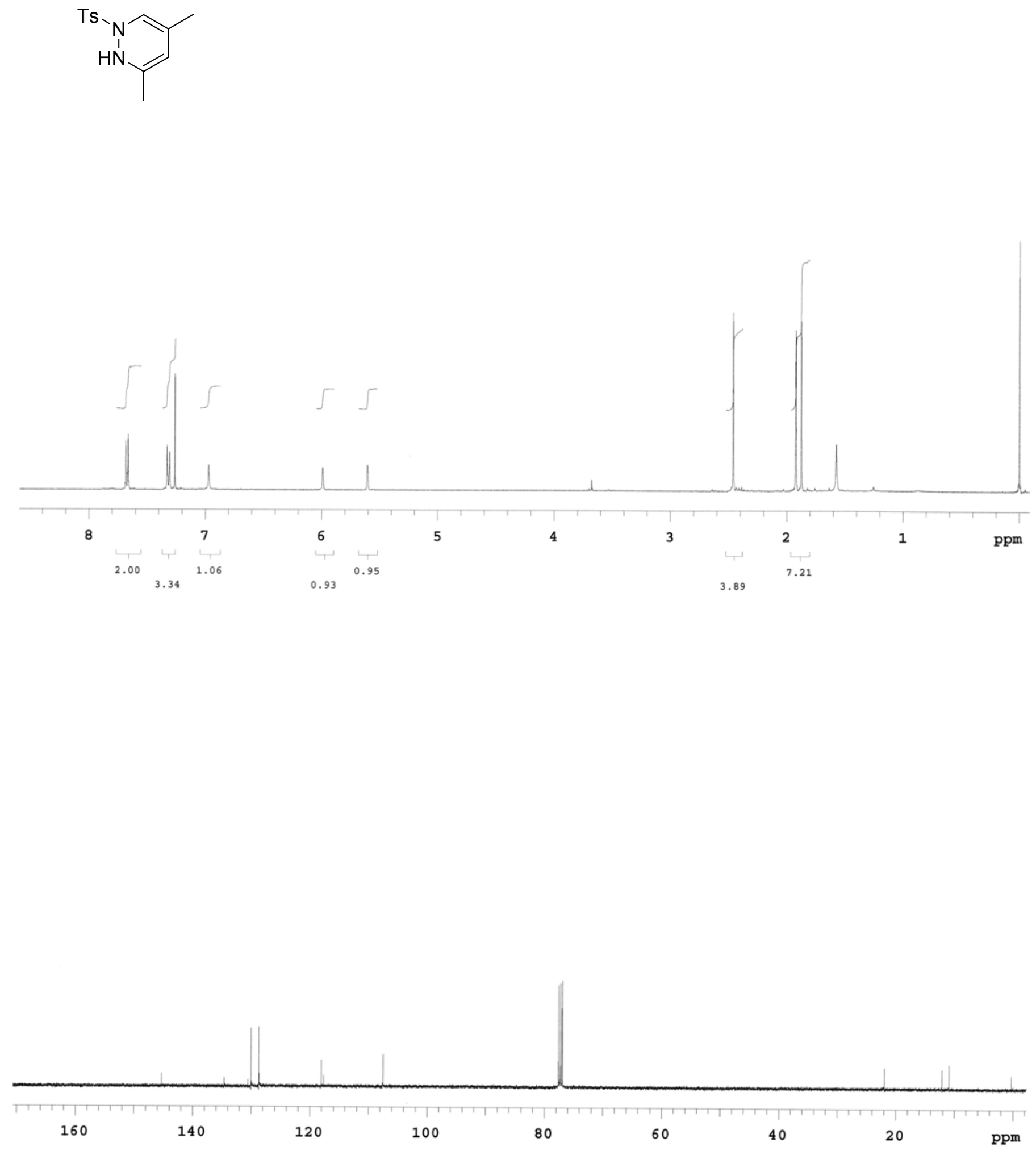

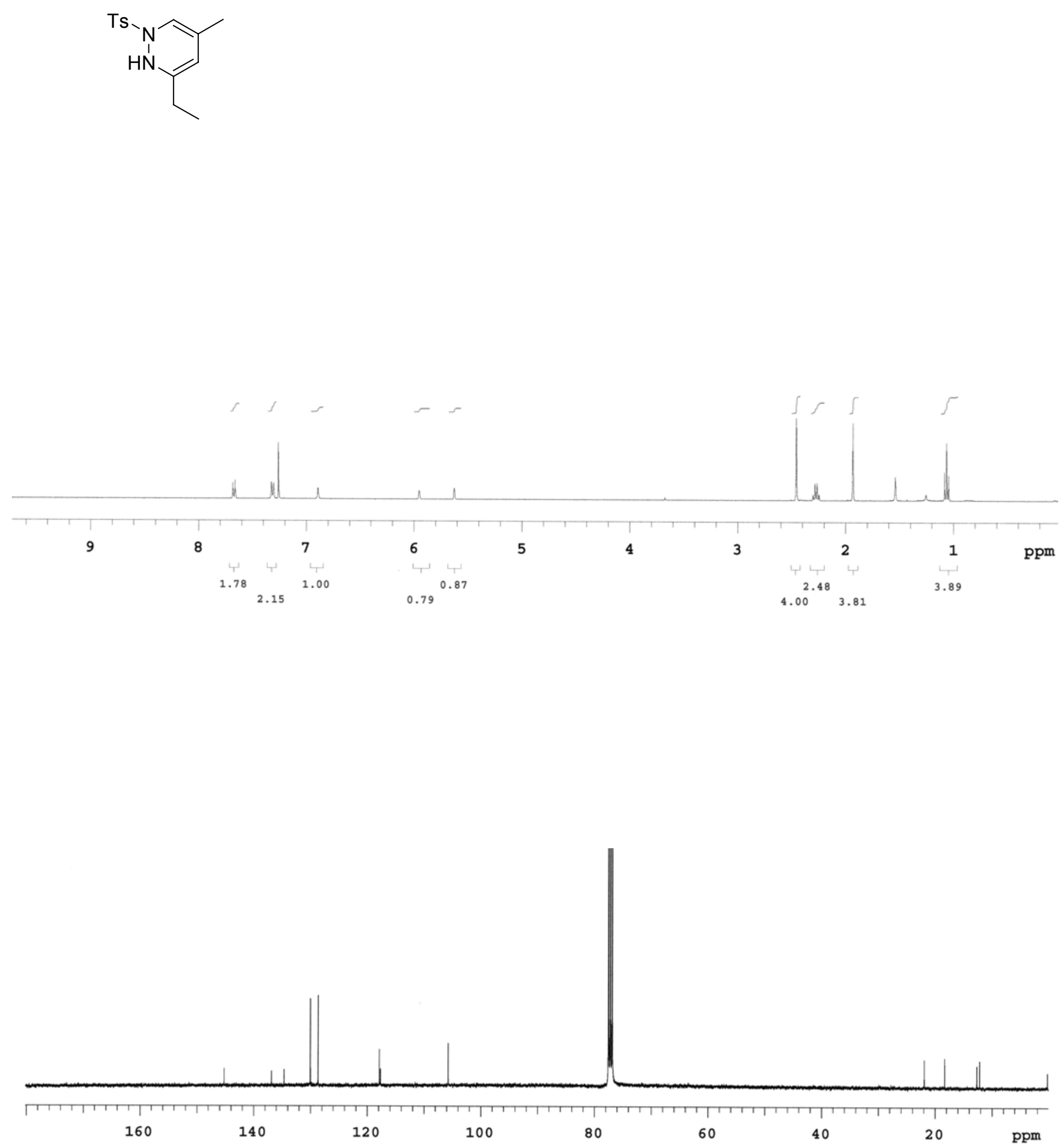

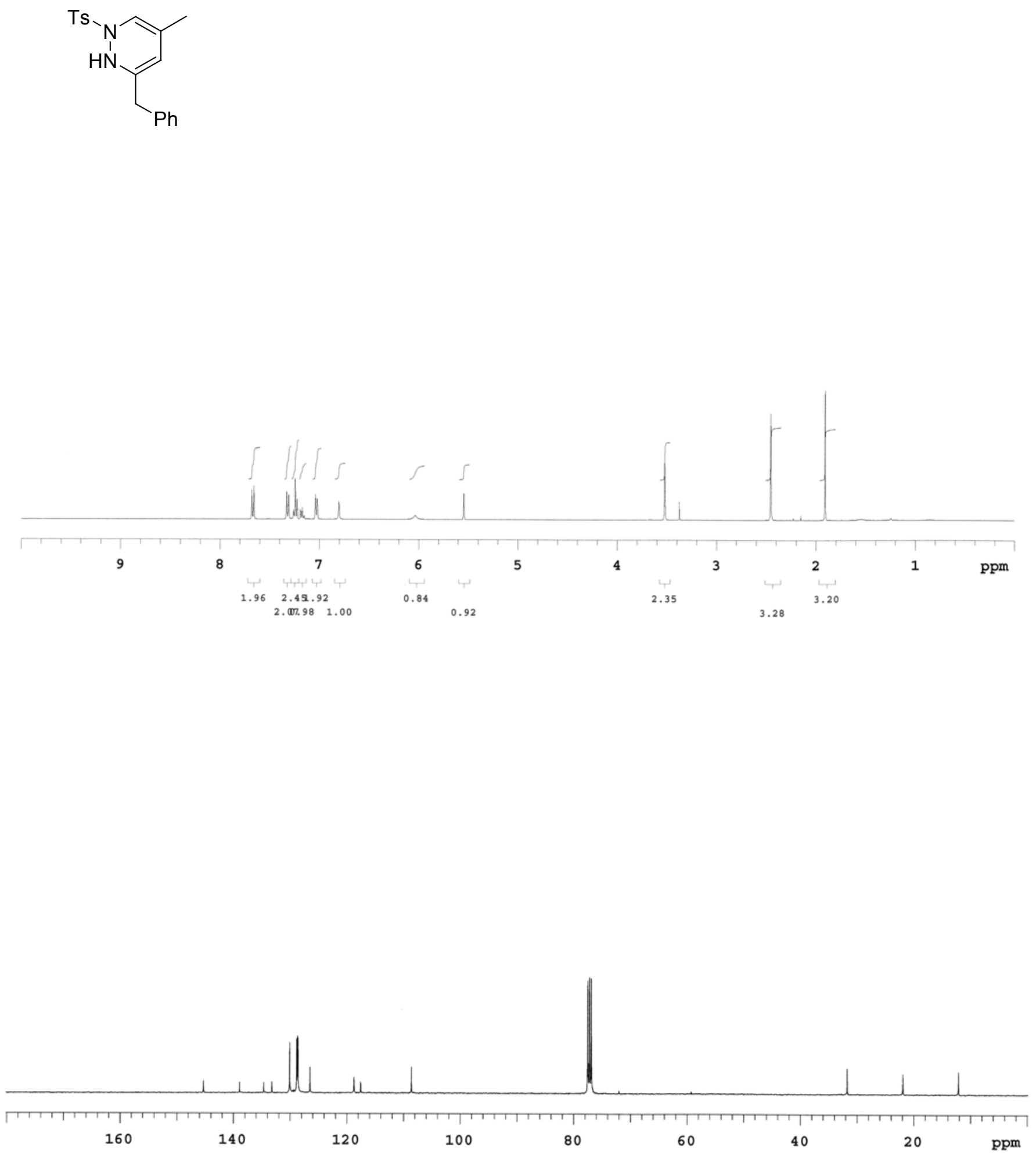

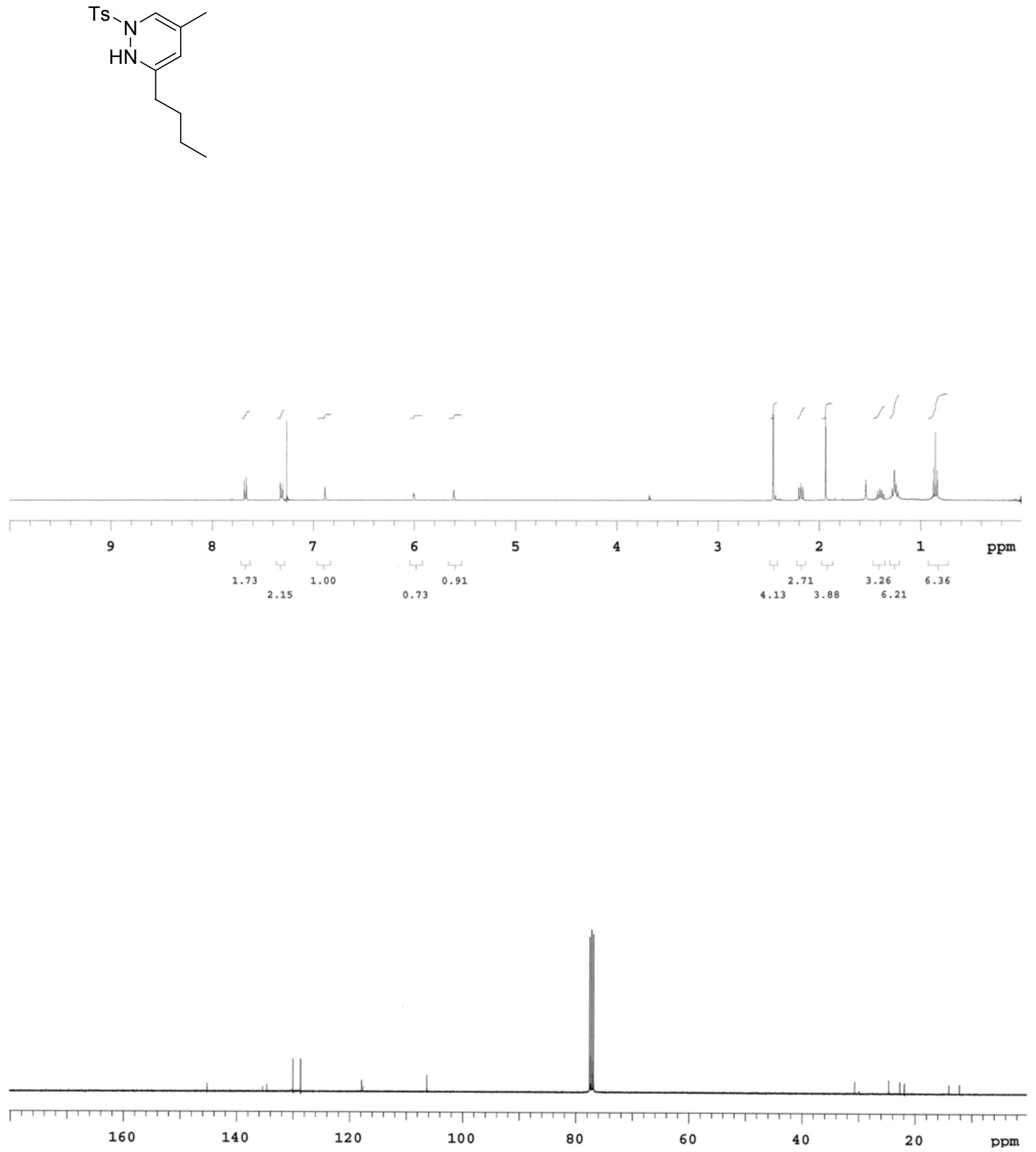


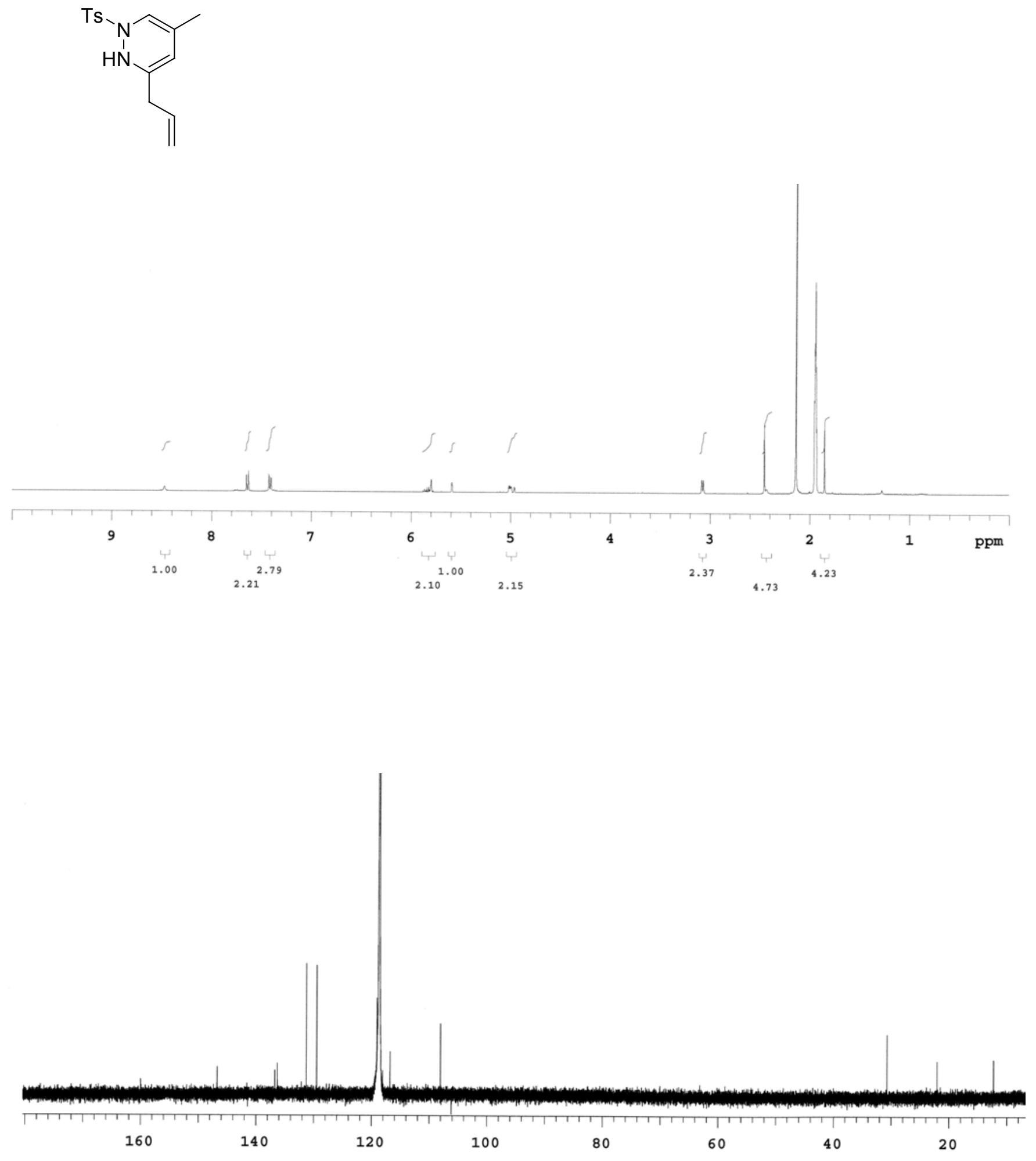



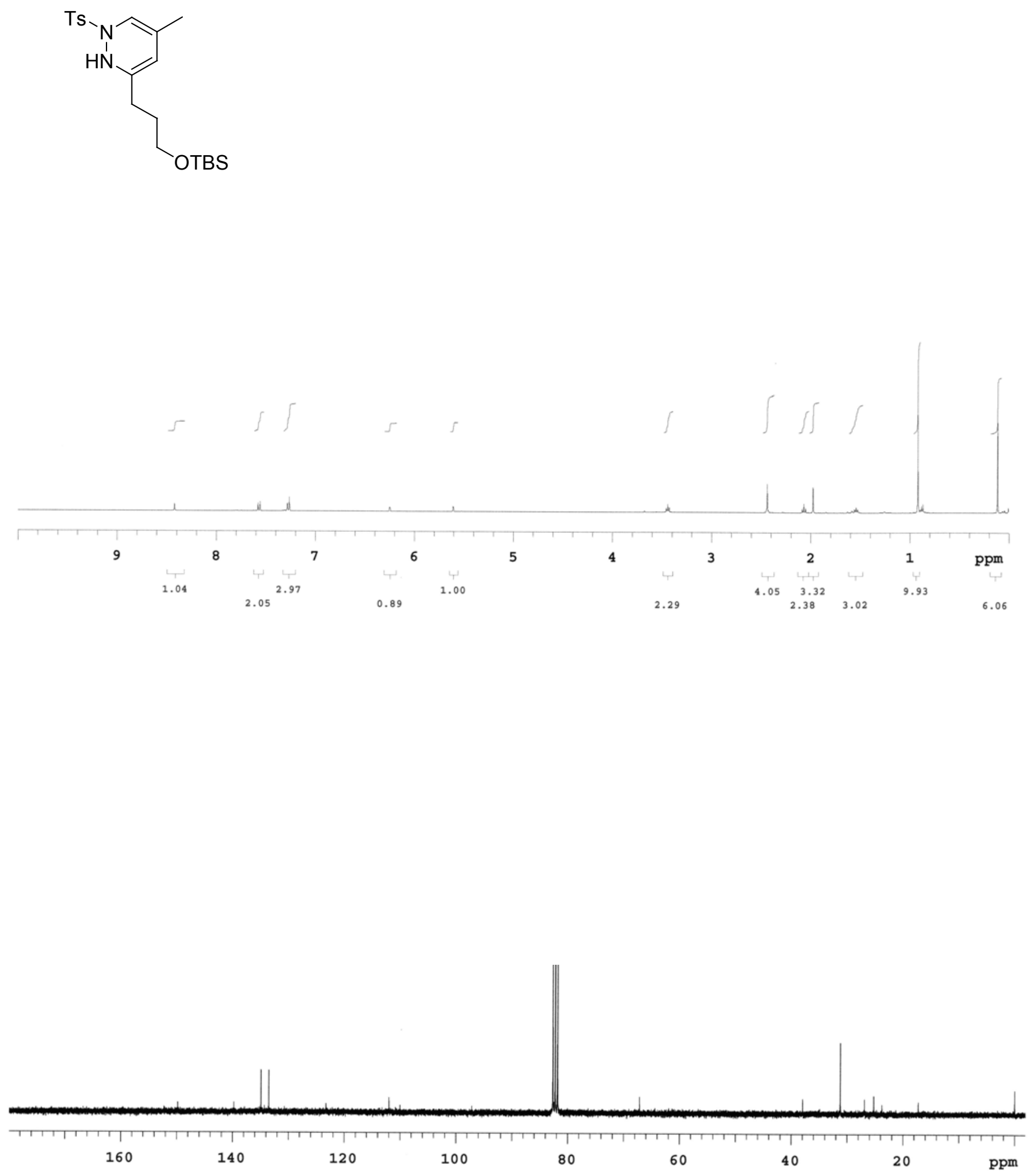
<smiles>CC1=CN([Te])NC(C2SCCCS2)=C1</smiles>
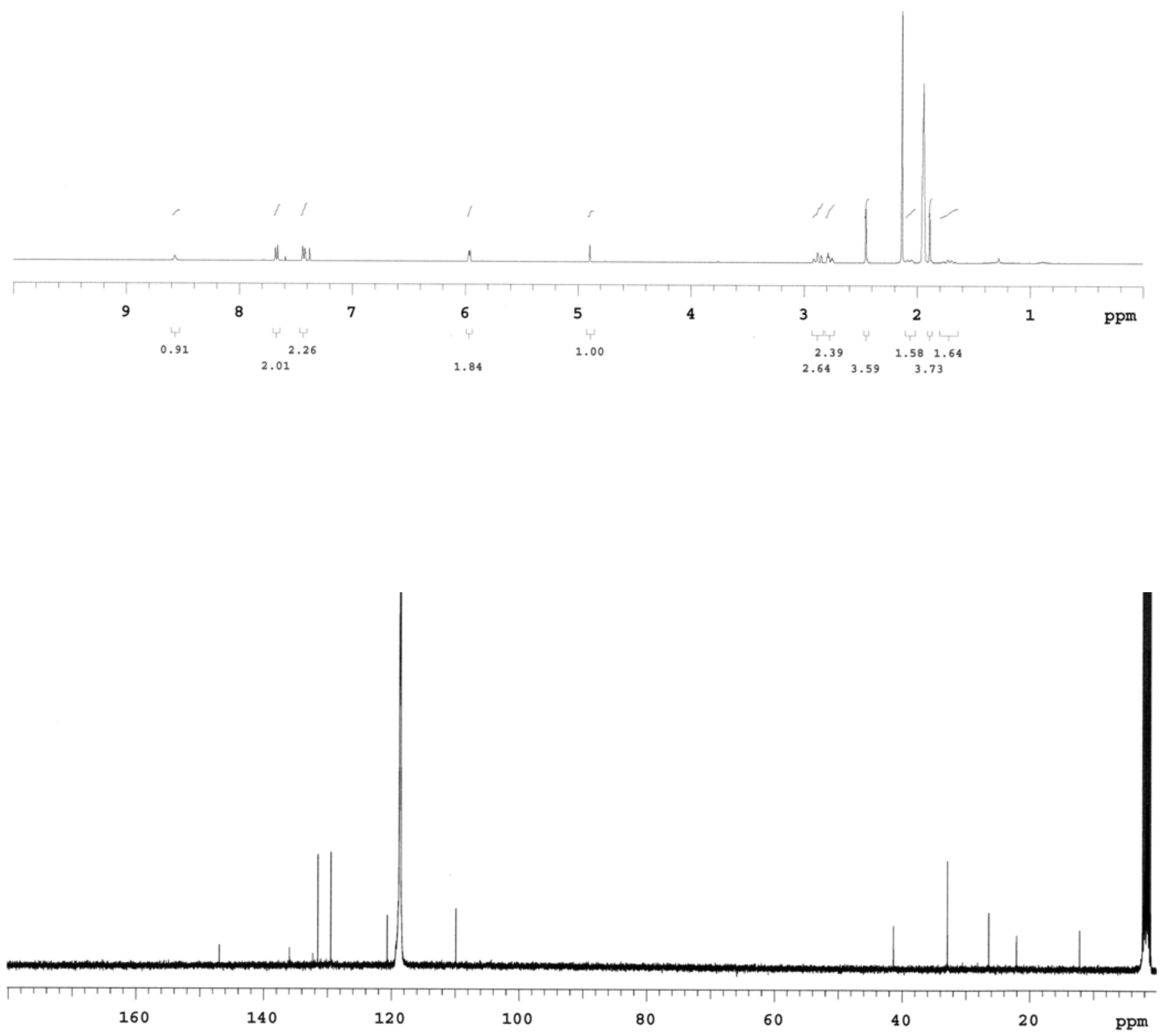

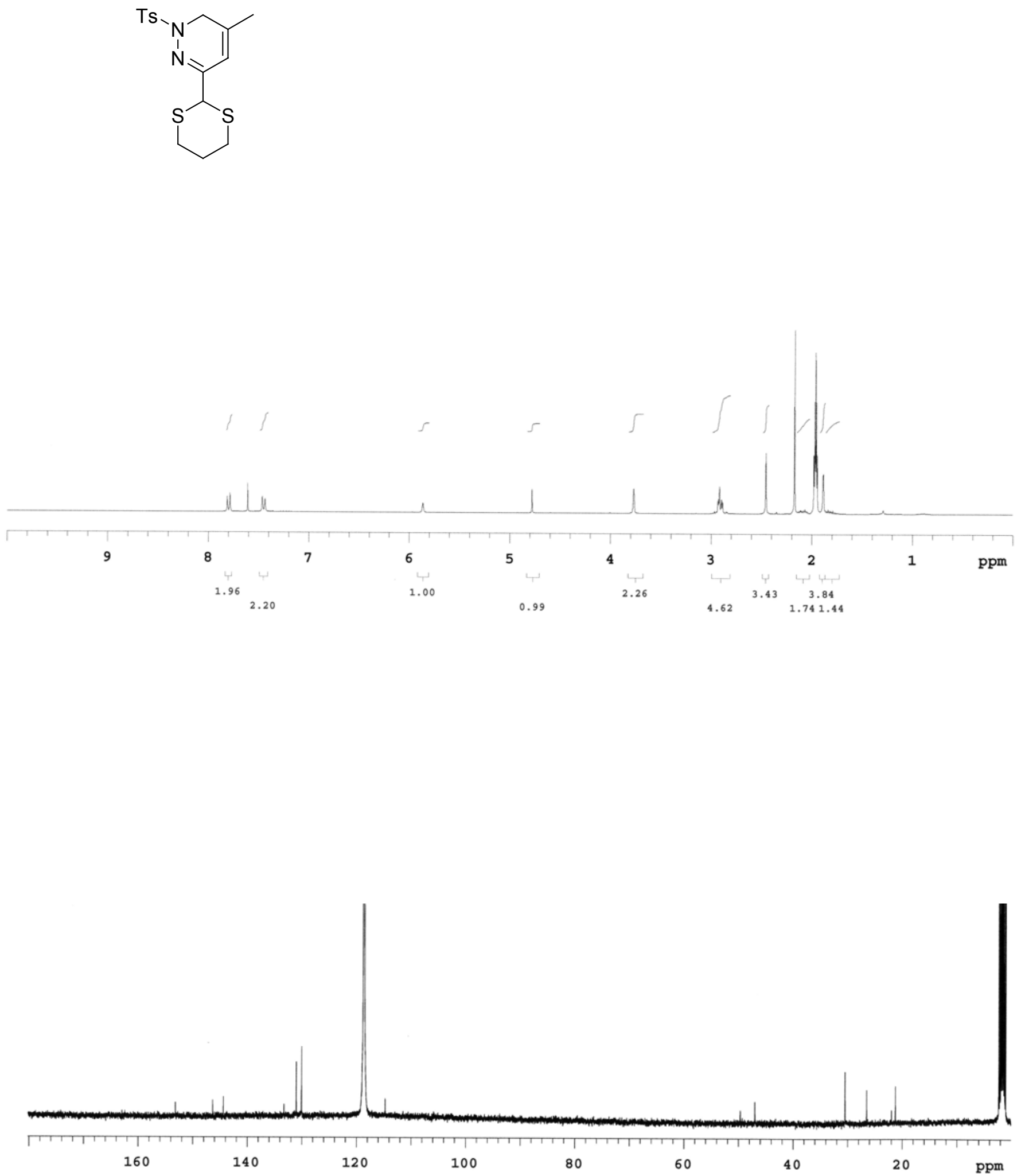

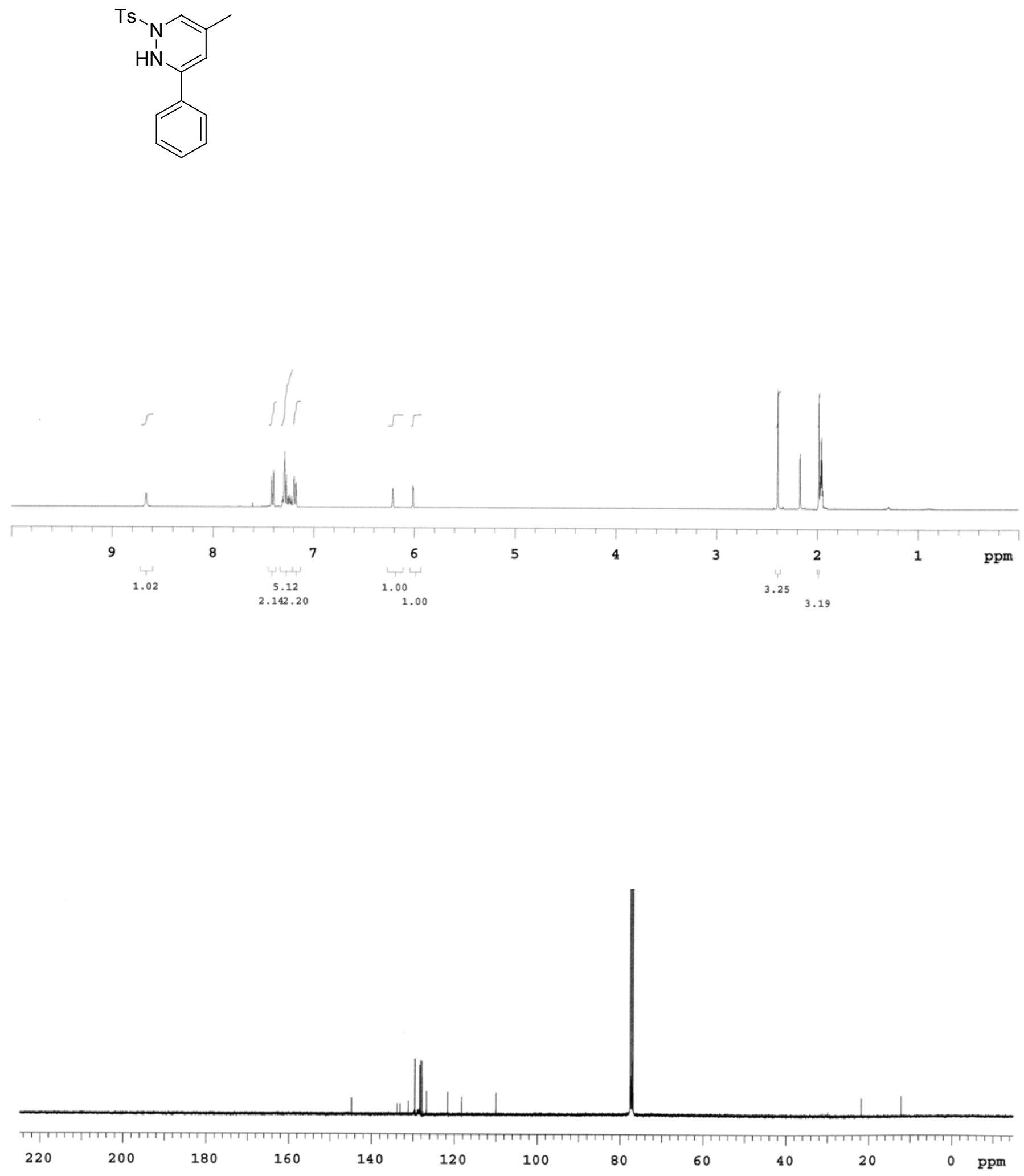

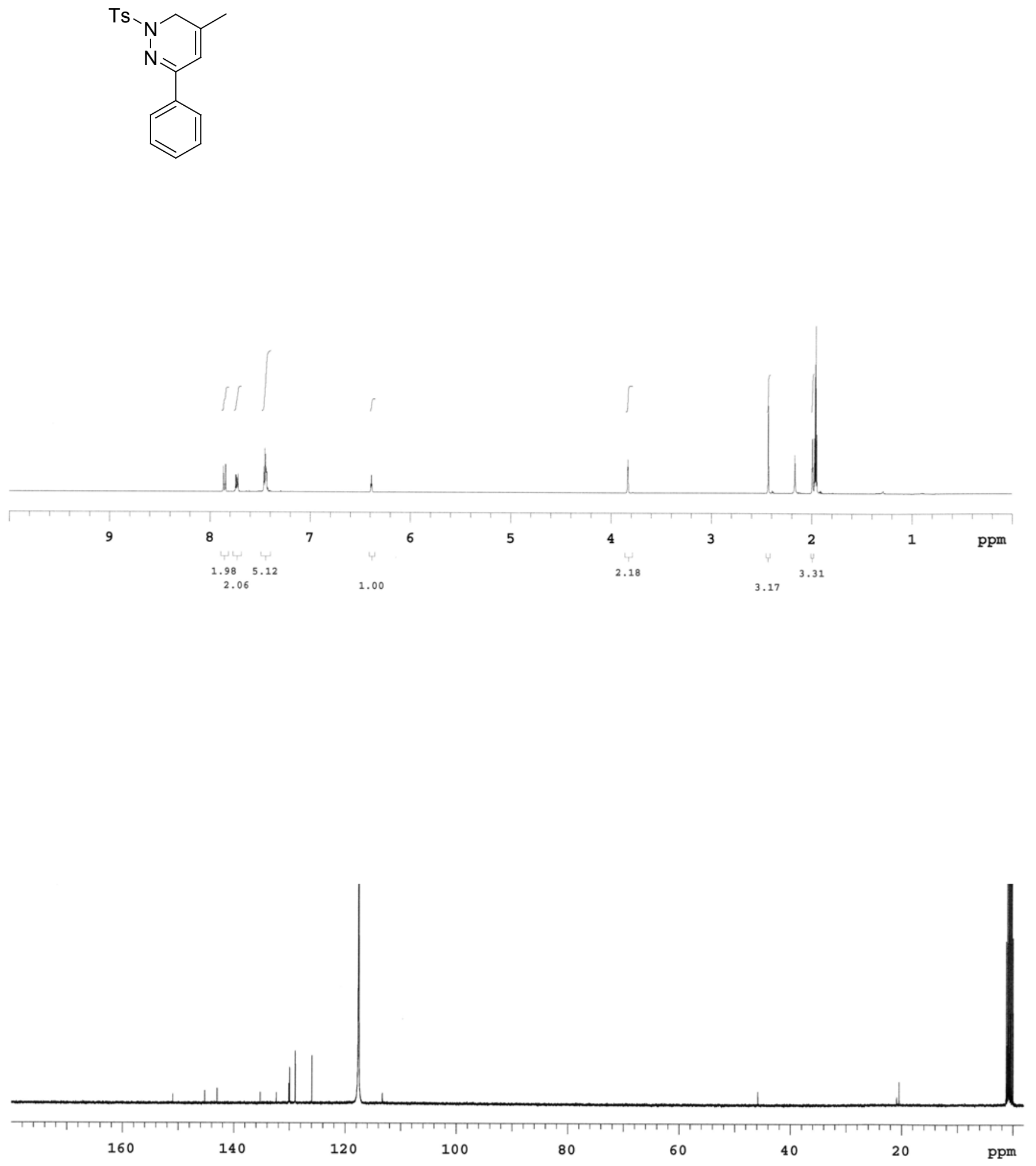

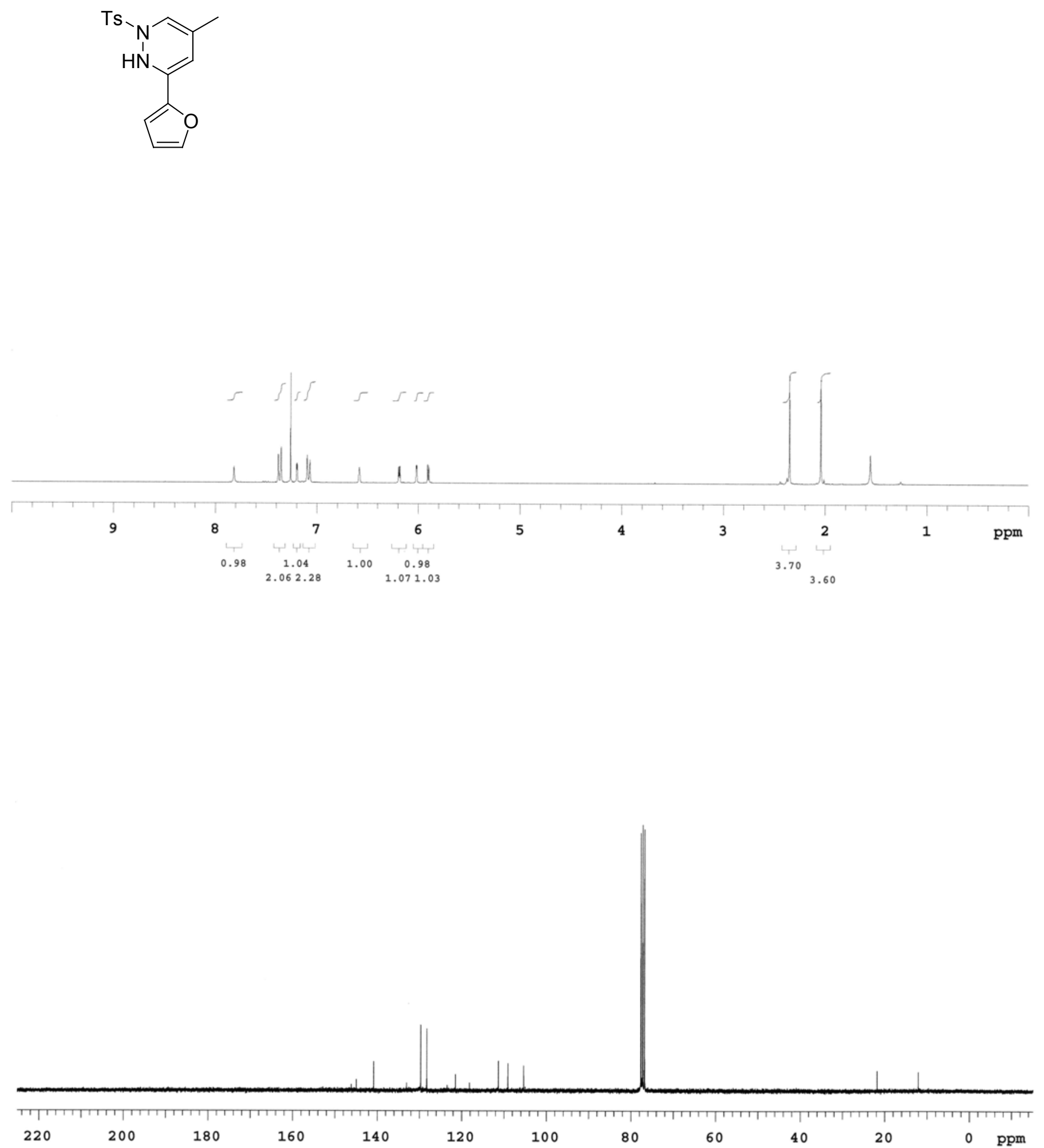

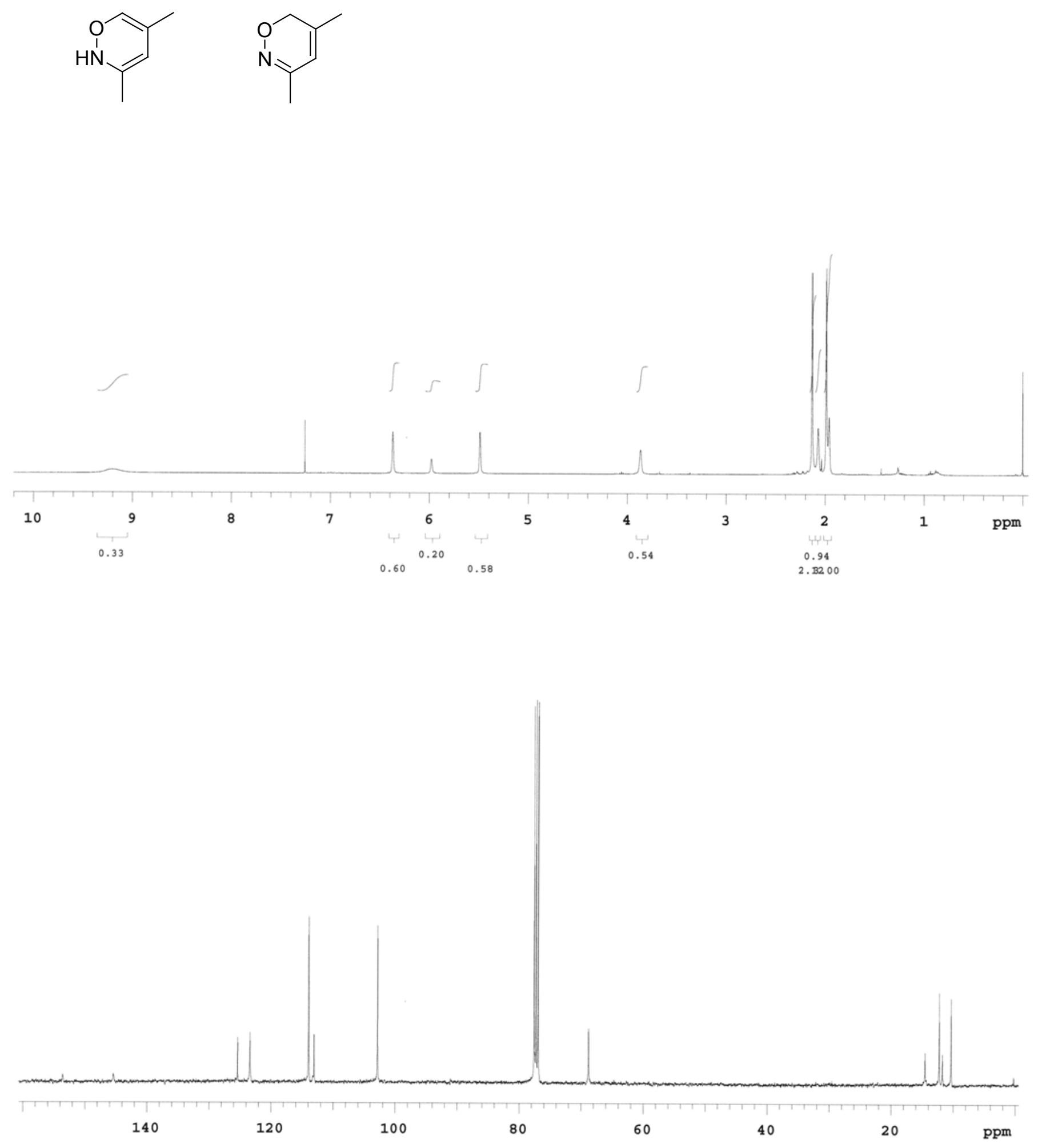

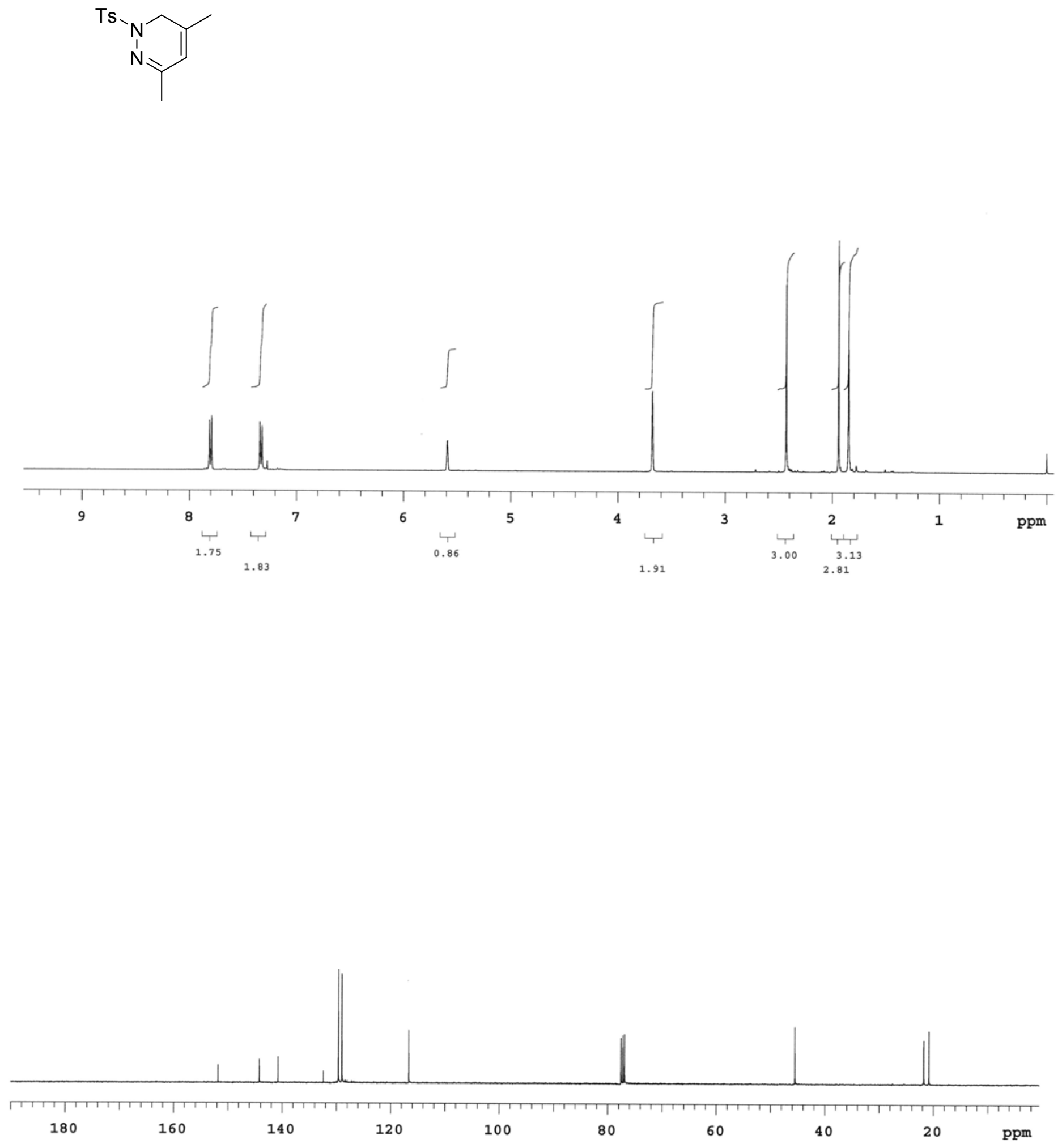

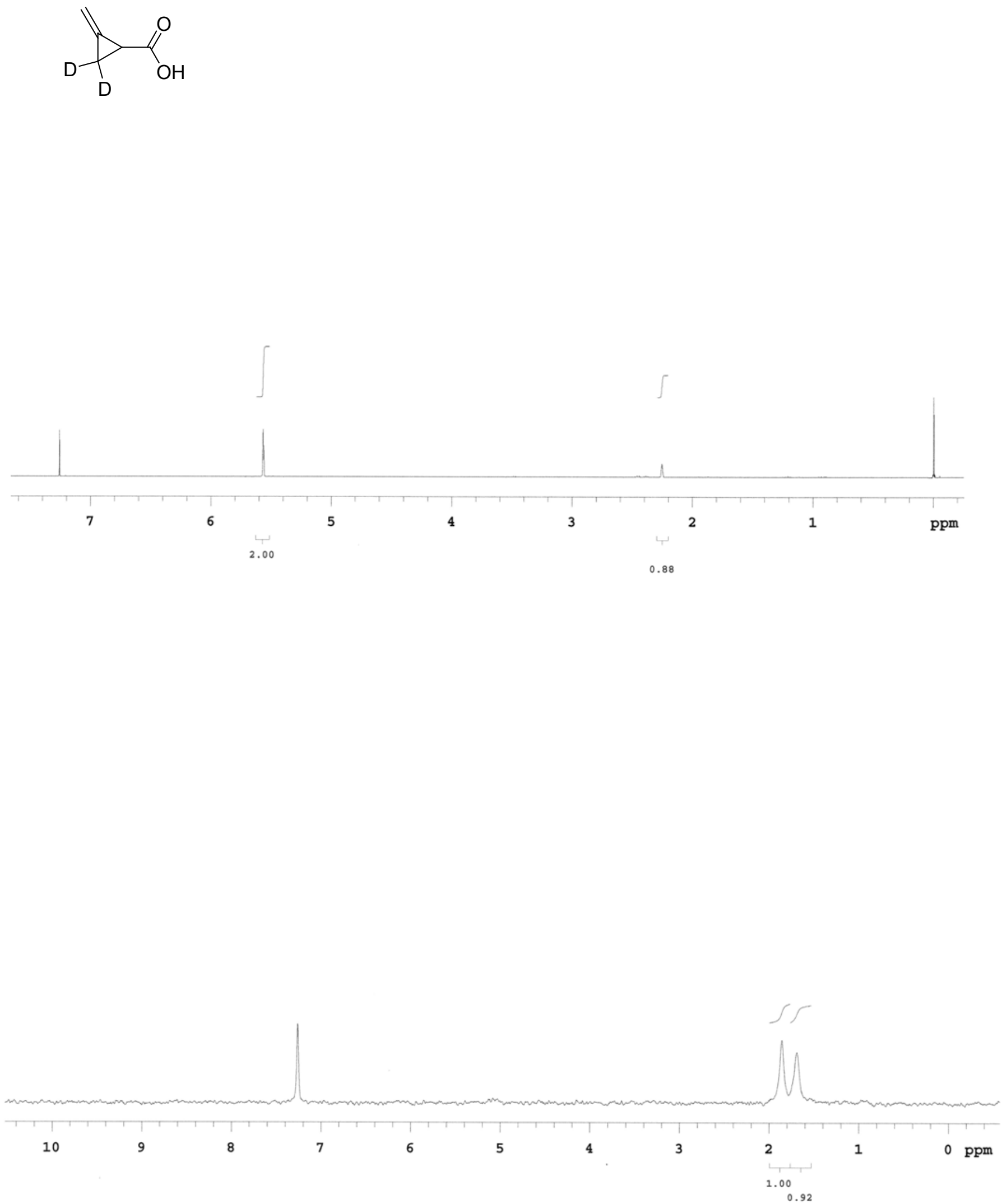

SI 42 

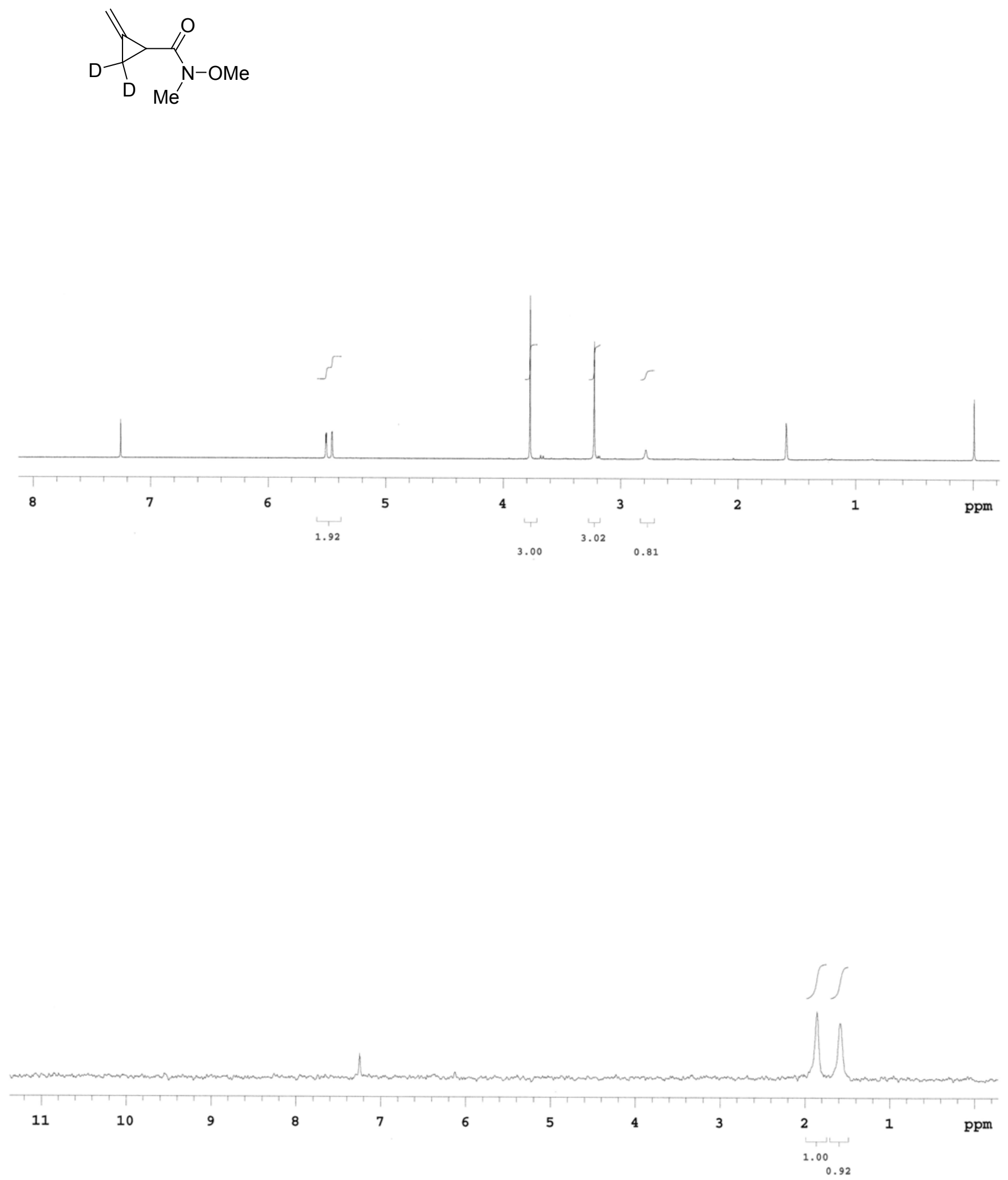

SI 43 

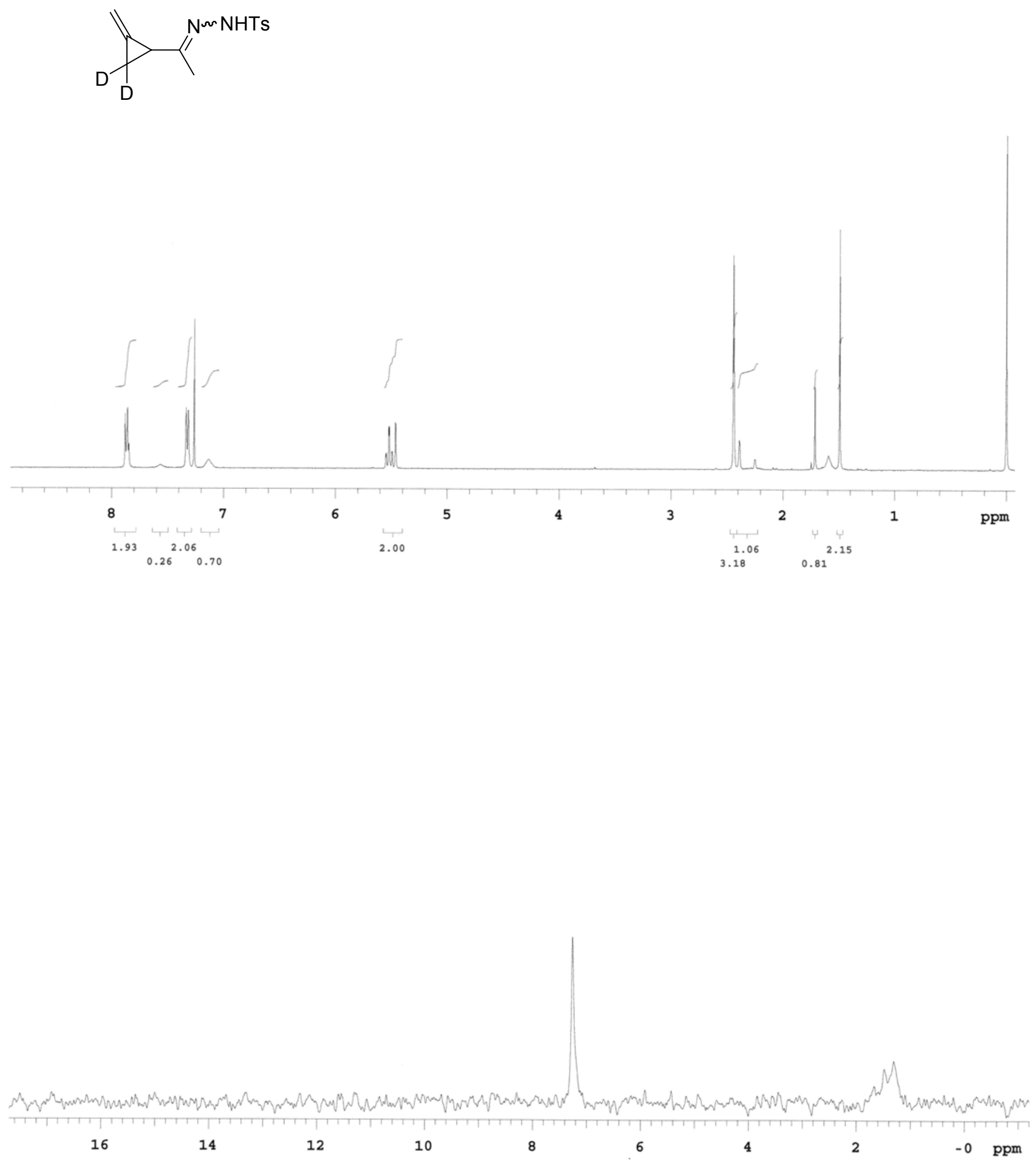

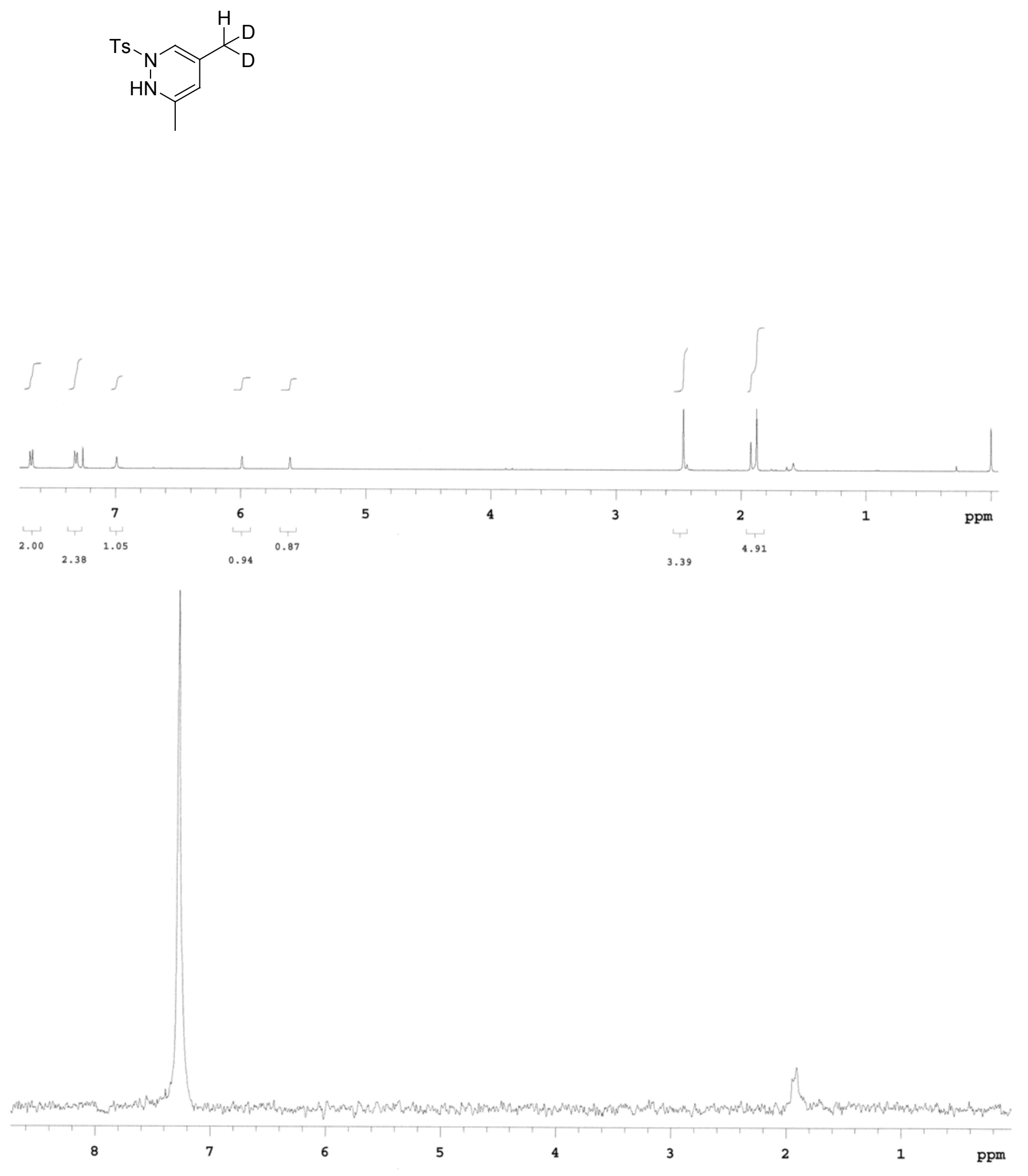


\section{References}

${ }^{1}$ Lai, M.-T.; Liu, L.-D.; Liu, H.-W. J. Am. Chem. Soc. 1991, 113, 7388.

${ }^{2}$ Winstein, S.; Friedrich, E. C.; Baker, R.; Lin, Y.-I. Tetrahedron Suppl. 1966, 8, 621.

${ }^{3}$ Molander, G. A.; Cormier, E. P. J. Org. Chem. 2005, 2622.

${ }^{4}$ Although this change in $\mathrm{E} / \mathrm{Z}$ isomer ratio upon crystallization was unexpected, such observations in other hydrazone and imine systems have been reported, see: a) Raban, M.; Carlson, E. J. Am. Chem. Soc. 1971, 93, 685. b) Bellamy, A. J.; Guthrie, R. D. J. Chem. Soc. C. 1968, 2090. c) Curtin, D. Y.; Hausser, J. W. J. Org. Chem. 1961, 83, 3474.

${ }^{5}$ a) Lautens, M.; Delanghe, P. H. M. J. Org. Chem. 1993, 58, 5037. b) Lautens, M.; Delanghe, P. H. M. J. Am. Chem. Soc. 1994, 116, 8526. c) Lautens, M.; Ren, Y. J. Org. Chem. 1996, 61, 2210 . 